University of New Mexico

UNM Digital Repository

Mathematics and Statistics Faculty and Staff

Publications

Academic Department Resources

2011

Evidence supporting measure of similarity for reducing the complexity in information fusion

Florentin Smarandache

Jean Dezert

Xinde Li

Xinhan Huang

Follow this and additional works at: https://digitalrepository.unm.edu/math_fsp

Part of the Data Storage Systems Commons, Digital Communications and Networking Commons, Mathematics Commons, Other Computer Engineering Commons, and the Robotics Commons 


\title{
Evidence supporting measure of similarity for reducing the complexity in information fusion is
}

\author{
Xinde $\mathrm{Li}^{\mathrm{a}}{ }^{\mathrm{a} *}$, Jean Dezert ${ }^{\mathrm{b}}$, Florentin Smarandache ${ }^{\mathrm{c}}$, Xinhan Huang ${ }^{\mathrm{d}}$ \\ ${ }^{a}$ Key Laboratory of Measurement and Control of CSE (School of Automation, Southeast University), Ministry of Education, Nanjing 210096, China \\ ${ }^{\mathrm{b}}$ ONERA (The French Aerospace Lab), 29 Av. de la Division Leclerc, 92320 Châtillon, France \\ ${ }^{\mathrm{c}}$ Chair of Mathematics and Sciences Department, University of New Mexico, 200 College Road, Gallup, NM 87301, USA \\ ${ }^{\mathrm{d}}$ Image Processing and Intelligent Control Key Laboratory of Education Ministry of China, Huazhong University of Science and Technology, Wuhan 430074, China
}

\section{A R T I C L E I N F O}

\section{Article history:}

Available online 28 October 2010

\section{Keywords:}

Information fusion

Belief function

Complexity reduction

Robot perception

$\mathrm{DSmT}$

Measure of similarity

Distance

Lattice

\begin{abstract}
A B S T R A C T
This paper presents a new method for reducing the number of sources of evidence to combine in order to reduce the complexity of the fusion processing. Such a complexity reduction is often required in many applications where the real-time constraint and limited computing resources are of prime importance. The basic idea consists in selecting, among all sources available, only a subset of sources of evidence to combine. The selection is based on an evidence supporting measure of similarity (ESMS) criterion which is an efficient generic tool for outlier sources identification and rejection. The ESMS between two sources of evidence can be defined using several measures of distance following different lattice structures. In this paper, we propose such four measures of distance for ESMS and we present in details the principle of Generalized Fusion Machine (GFM). Then we apply it experimentally to the real-time perception of the environment with a mobile robot using sonar sensors. A comparative analysis of results is done and presented in the last part of this paper.
\end{abstract}

(c) 2010 Elsevier Inc. All rights reserved.

\section{Introduction}

Information fusion (IF) has gained more and more interests in the scientific community since the end of the 90s with the development of sophisticated multi-sensor and hybrid (involving human feedbacks in the loop) systems in many fields of applications (such as robotics, defense, security, medicine). IF appears through many scientific international conferences and workshops [10]. The main theories useful for information fusion are Probability Theory [18,28] (and more recently Imprecise Probability Theory [41]), Possibility Theory [7] (based on Fuzzy Sets Theory [48]), Neutrosophic Set Theory [17] and belief function theories (such as Dempster-Shafer Theory (DST) [32] and more recently Dezert-Smarandache Theory (DSmT) [34-36]).

In this work, we concentrate our attention on belief function theories and specially on DSmT because of its ability to deal efficiently with uncertain, imprecise, conflicting, quantitative and qualitative information. Basically, in DST, a basic belief assignment (bba) $m(\cdot)$ is a mapping from the power set $2^{\Theta}$ (see Section 2.2 for details) of the frame of discernment $\Theta$ into $[0,1]$ such that

\footnotetext{
This work was supported by National Natural Science Foundation of China (Nos. 60804063 and 60805032), Natural Science Foundation of Jiangsu Province under Grant (No. BK2010403), The Public Funds of Image Processing and Intelligent Control Key Laboratory of Chinese Education Ministry under Grant (No. 200902), The Science and Technology Innovation Foundation in Southeast University under Grant (No. 3208000501) and Aeronautical Science Foundation of China under Grant (No. 20100169001).

* Corresponding author.

E-mail addresses: xindeli@seu.edu.cn (X. Li), jean.dezert@onera.fr (J. Dezert), smarand@unm.edu (F. Smarandache), xhhuang@mail.hust.edu.cn (X. Huang).
} 


$$
m(\emptyset)=0 \quad \text { and } \quad \sum_{X \in 2^{\Theta}} m(X)=1 .
$$

In DST, $\Theta$ represents the set of exclusive and exhaustive possibilities for the solution of the problem under consideration. In DSmT, $\Theta$ can be a set of possible nonexclusive elements and the definition of bba is extended to the lattice structures of hyper-power set $D^{\Theta}$ (see also Section 2.3 for a brief presentation and $[6,9,36]$ for definitions, details and examples), and to super-power set $S^{\Theta}$ in UFT (Unification of Fusion Theories) [33,35]. In general, $m(\cdot)$ is not a measure of probability, except in the case when its focal elements (that is, the elements which have a strictly positive mass of belief) are singletons; in such case, $m(\cdot)$ is called a Bayesian bba [32] which can be considered as a subjective probability measure. In belief function theories, the main information fusion problem consists in finding an efficient way for combining several sources of evidence $s_{1}, s_{2}, \ldots, s_{n}$ characterized by their basic belief assignments (bba's) $m_{1}(\cdot), m_{2}(\cdot), \ldots, m_{n}(\cdot)$. These bba's are defined on the same fusion space, either $2^{\Theta}, D^{\Theta}$, or $S^{\Theta}$ depending on the underlying model associated with the nature of the frame $\Theta$. The difficulty in information fusion arises from the fact that the sources can be conflicting (that is, one source assigns some beliefs to a proposition $A$ whereas another source assigns some beliefs to a proposition $B$, but $A$ and $B$ are known to be truly exclusive $(A \cap B=\emptyset))$ and one needs a solution for dealing with conflicting information in the fusion. In DST, Shafer proposes Dempster's rule of combination, as the fusion operator, to combine sources of evidence whereas in DSmT the recommended fusion operator is PCR5 (Proportional Conflict Redistribution \# 5) rule of combination, see [32] and [36] for discussions and comparisons of these rules. PCR5 is more complex than Demspter's rule but it offers a better ability to deal with conflicting information.

However, both rules become intractable in some applications having only low computational capacities (as in some autonomous onboard systems by example) because their complexity increases drastically with the number $n$ of sources combined or with the size of the frame $\Theta$, specially in the worst case (that is, when a strictly positive mass of belief is assigned to all elements of the fusion space). To avoid this problem, one can do: (1) to reduce the number of sources combined and (2) to reduce the size of the frame $\Theta$. In this paper, we propose a solution only for reducing the number of sources combined. We are not concerned about the second aspect in our application of robot perception since in this application our frame $\Theta$ has only two elements representing the emptiness or occupancy states of the grid cells of the sensed map of the environment. To expect good performances of such a limited-resource fusion scheme, it seems natural to search and combine altogether only the sources which are coherent (which are not too conflicting) according to a given measure of similarity in terms of Dedekind's lattice.

Such an idea has been already investigated by several authors who have proposed some distance measures between two evidential sources in different fields of applications. For example, Tessem [38], in 1993, proposed the distance $d_{i j}=\max _{\theta_{1} \in \Theta}\left|\operatorname{Bet} P_{i}\left(\theta_{l}\right)-\operatorname{Bet}_{j}\left(\theta_{l}\right)\right|$ according to the pignistic probability transform $\operatorname{Bet} P(\cdot)$. In 1997, Bauer [1] introduced two other measures of error to take a decision based on pignistic probability distribution after approximation. In 1998, Zouhal and Denoeux [49] also introduced a distance based on mean square error between pignistic probability. In 1999, Petit-Renaud [29] defined a measure directly on the power set of $\Theta$ and proposed an error criterion between two belief structures based on the generalized Hausdorff distance. In 2001, Jousselme et al. [13] proposed in DST framework, a new distance measure $d_{i j}=1-\frac{1}{\sqrt{2}} \sqrt{m_{1}^{2}+m_{2}^{2}-2\left\langle m_{1}, m_{2}\right\rangle}$ between two bba's for measuring their similarity (closeness). In 2006, Ristic and Smets [30,31] defined in the TBM (Transferable Belief Model) framework a TBM-distance between bba's to solve the association of uncertain combat ID declarations. These authors also recalled Bhattacharya's distance $d_{i j}=\sqrt{1-\sum_{A \in F_{i}} \sum_{B \in F_{j}} \sqrt{m_{i}(A) m_{j}(B)}}$ between two bba's. In 2006, Diaz et al. [5] proposed a new measure of similarity between bba's based on Tversky's similarity measure [40]. Note that in belief function theories, the classical measures used in Probability Theory (for example, Kullback-Leibler's (KL) distance [2]) couldnot be applied directly because bba's werenot probability measures in general. Kaburlasos et al. [16,19] also proposed several valuable similarity measure functions based on an inclusion measure $[14,15]$ and a metric respectively. Dongrui $\mathrm{Wu}$ et al. proposed a vector similarity measure for linguistic approximation, which was very useful in understanding the uncertainties associated with linguistic terms [45]. And then, he also compared deeply ranking methods, similarity measures and uncertainty measures for interval type-2 fuzzy sets [46].

In this paper, we develop an Evidence Support Measure of Similarity (ESMS) in a generalized fusion space according to different lattices structures $[6,9,47]$ for reducing the number of sources of evidence to combine. This allows us to reduce drastically the computational complexity of the fusion processing for the real-time implementation purpose. As shown in the next sections, we propose several possible measures of distance for ESMS. The purpose of this paper is neither to select, nor to justify, the best measure of distance for ESMS but only to illustrate from a real-time experiment with real data the advantages of this new approach.

This paper is organized as follows. In Section 2, we briefly recall the main paradigms for dealing with uncertain information. In Section 3, we give a general mathematical definition of ESMS between two basic belief assignments and some basic properties of ESMS. In Section 4, we extend and present different possible ESMS functions (distance measures) fitting with the different lattice structures listed in Section 2. A comparison of the performances of four possible distances is made through a simple example in Section 5. The simulation presented in Section 6 shows in details how ESMS filter is used within GFM scheme and its advantage. An application of ESMS filter in GFM for mobile robot perception with real-data (sonar sensors measurements) is presented in Section 7 to show the advantages of the approach proposed here. The conclusion is given in Section 8. 


\section{The main paradigms for dealing with uncertainties}

\subsection{Probability Theory and Bayesian rule}

Probability Theory [18] is the original theory for dealing with uncertainty. We will not present this theory in detail since there exist dozens of classical books devoted to it, see for example [28]. We just recall that a random experiment is an experiment (action) whose result is uncertain before it is performed and a trial is a single performance of the random experiment. An outcome is the result of a trial and the sample space $\Theta$ is the set of all possible outcomes of the random experiment. An event is the subset of the sample space $\Theta$ to which a probability measure can be assigned. Two events $A_{i}$ and $A_{j}$ are said exclusive (disjoint) if $A_{i} \cap A_{j}=\emptyset, \forall i \neq j$, where the empty set $\emptyset$ represents the impossible event. The sure event is the sample space $\Theta$. Probability Theory is based on Set Theory and the measure theory on sets. The following axioms have been identified as the necessary and sufficient condition for probability $P(\cdot)$ as a measure: Axiom ( 1$)$ (nonnegativity) $0 \leqslant P(A) \leqslant 1$, Axiom (2) (unity) $P(\Theta)=1$, and Axiom (3) (finite additivity, ${ }^{1}$ ) if $A_{1}, A_{2}, \ldots, A_{n}$ are disjoint events, then $P\left(A_{1} \cup A_{2} \cup \ldots \cup A_{n}\right)=$ $\sum_{i=1}^{n} P\left(A_{i}\right)$. Events which are subsets of the sample space are put in one-to-one correspondence with propositions in belief function theory [32] (pages 35-37). That's why we use indifferently the same terminology for set, event or proposition in this paper. The probabilistic inference is (usually) carried out according to Bayesian rule:

$$
\forall B, P(B)>0, \quad P\left(A_{i} \mid B\right)=\frac{P\left(A_{i} \cap B\right)}{P(B)}=\frac{P\left(B \mid A_{i}\right) P\left(A_{i}\right)}{\sum_{j=1}^{n} P\left(B \mid A_{j}\right) P\left(A_{j}\right)},
$$

where the sample space $\Theta$ has been partitioned into exhaustive and exclusive events $A_{1}, A_{2}, \ldots, A_{n}$, that is, $A_{i} \cap A_{j}=\emptyset,(i \neq j)$ and $A_{1} \cup A_{2} \cup \cdots \cup A_{n}=\Theta ; P(\cdot)$ is an a priori probability measure defined on $\Theta$ satisfying Kolmogorov's axioms. In Bayesian formula, it is assumed that the denominator is strictly positive. A generalization of this rule has been proposed by Jeffrey $[11,12]$ for working in circumstances where the parochialist assumption is not a reasonable assumption, i.e. when $P(B \mid B)=1$ is a fallacy, see $[12,25]$ for details and examples.

Using the classical terminology adopted in belief function theories (DST or DSmT) and considering for example $\Theta=\{A, B\}$, a discrete probability measure $P(\cdot)$ can be interpreted as a specific Bayesian belief mass $m(\cdot)$ such that

$$
m(A)+m(B)=1 .
$$

\subsection{Dempster-Shafer Theory (DST)}

In DST [32], the frame of discernment $\Theta$ of the fusion problem under consideration consists in a discrete finite set of $n$ exhaustive and exclusive elementary hypotheses $\theta_{i}$, that is, $\Theta=\left\{\theta_{1}, \theta_{2}, \ldots, \theta_{n}\right\}$. This is called Shafer's model of the problem. Such model assumes that an ultimate refinement of the problem is possible, exists and is achievable, so that elements $\theta_{i}$, $i=1,2, \ldots, n$ are precisely defined and identified in such a way that we are sure that they are truly exclusive and exhaustive (closed-world assumption). The set of all subsets of $\Theta$ is called the power set of $\Theta$ and is denoted $2^{\Theta}$. Its cardinality is $2^{|\Theta|}$. Since $2^{\Theta}$ is closed under $\cup$ and all $\theta_{i}, i=1,2, \ldots, n$ are exclusive, it defines a Boolean algebra. All composite propositions built from elements of $\Theta$ with $\cup$ operator such that:

(1) $\emptyset, \theta_{1}, \ldots, \theta_{n} \in 2^{\Theta}$;

(2) If $A, B \in 2^{\Theta}$, then $A \cup B \in 2^{\Theta}$;

(3) No other elements belong to $2^{\Theta}$, except those obtained by using rules (1) or (2).

Shafer defines a basic belief assignment (bba), also called mass function, as a mapping $m(\cdot): 2^{\Theta} \rightarrow[0,1]$ satisfying $m(\emptyset)=0$ and the normalization condition. Typically, when $\Theta=\{A, B\}$ and Shafer's model holds, in DST one works with $m(\cdot)$ such that

$$
m(A)+m(B)+m(A \cup B)=1,
$$

$m(A \cup B)$ allows us to assign some beliefs to the disjunction $A \cup B$ which represents the ignorance in choosing either $A$ or $B$. From this simple example, one clearly sees the ability of DST in offering a better model for a total ignorant/vacuous source of information which is obtained by setting $m(A \cup B)=1$. For Probability Theory, one would be forced to adopt the principle of insufficient reason (also called the principle of indifference) to take $m(A)=m(B)=1 / 2$ as default belief mass for representing a total ignorant body of evidence.

In DST framework, the combination of two belief assignments $m_{1}(\cdot)$ and $m_{2}(\cdot)$ is done with Dempster's rule of combination. This rule is nothing but a normalized version of the conjunctive rule in order to remove the total conflicting mass [32]. Dempster's rule is defined if and only if the two sources of evidence are not fully conflicting, that is, when $\sum_{X_{1}, X_{2} \in 2^{\Theta}} m_{1}\left(X_{1}\right) m_{2}\left(X_{2}\right) \neq 1$ and it is mathematically defined by $m(\emptyset)=0$ and for $X \neq \emptyset$ by

$X_{1} \cap X_{2}=\emptyset$

\footnotetext{
1 Another axiom related to the countable additivity can be also considered as the fourth axiom of Probability Theory.
} 


$$
m(X)=\frac{\sum_{\substack{X_{1}, X_{2} \in 2^{\Theta} \\ X_{1} \cap X_{2}=X}} m_{1}\left(X_{1}\right) m_{2}\left(X_{2}\right)}{1-\sum_{\substack{X_{1}, X_{2} \in 2^{\Theta} \\ X_{1} \cap X_{2}=\emptyset}} m_{1}\left(X_{1}\right) m_{2}\left(X_{2}\right)} .
$$

\subsection{Dezert-Smarandache Theory (DSmT)}

In DSmT framework [34-36], the frame $\Theta=\left\{\theta_{1}, \theta_{2}, \ldots, \theta_{n}\right\}$ is a finite set of $n$ exhaustive elements which are not necessarily exclusive. The principle of the third excluded middle and Shafer's model are refuted in DSmT (but can be introduced if needed depending on the model of the frame one wants to deal with). For a wide class of fusion problems, this is because the nature of hypotheses can be only a vague and imprecise or a crude approximation of the reality and no ultimate refinement is achievable. As a simple example, if we consider two suspects, $\operatorname{Peter}(P)$ and Mary $(M)$, in some criminal investigations, it is possible that Peter has committed the crime alone, as well as Mary, or maybe Peter and Mary have committed the crime together. In that case, one has to consider the possibility for $P \cap M \neq \emptyset$, but there is no way to refine the original frame $\Theta=\{P, M\}$ into a finer one with exclusive finer elements, i.e. $\Theta^{\prime}=\{P \backslash(P \cap M), P \cap M, M \backslash(P \cap M)\}$, because there is no physical meaning and no possible occurrence of the atomic granules $P \backslash(P \cap M)$ and $M \backslash(P \cap M)$. In other words, the exclusive elements of the refined frame satisfying Shafer's model cannot always be well identified and precisely separated or may make no sense at all. This is why DSmT deals with nonexclusive, partially overlapped or vague elements and refutes Shafer's model and third excluded middle assumptions. DSmT proposes to work in a fusion space defined by Dedekind's lattice also called hyper-power set $D^{\Theta}$.

The hyper-power set is defined as the set of all composite propositions built from elements of $\Theta$ with $\cap$ and $\cup$ operators such that [4]:

(1) $\emptyset, \theta_{1}, \ldots, \theta_{n} \in D^{\Theta}$;

(2) If $A, B \in D^{\Theta}$, then $A \cup B \in D^{\Theta}$ and $A \cap B \in D^{\Theta}$;

(3) No other elements belong to $D^{\Theta}$, except those obtained by using rules (1) or (2).

Following Shafer's idea, Dezert and Smarandache define a (generalized) basic belief assignment (or mass) as a mapping $m(\cdot): D^{\Theta} \rightarrow[0,1]$ such that:

$$
m(\emptyset)=0 \text { and } \sum_{X \in D^{\Theta}} m(X)=1
$$

Typically, when $\Theta=\{A, B\}$ and Shafer's model doesnot hold, in DSmT one works with $m(\cdot)$ such that

$$
m(A)+m(B)+m(A \cup B)+m(A \cap B)=1
$$

which appears actually as a direct and natural extension of (2) and (3).

Actually DSmT also offers an advantage to work with Shafer's model or with any hybrid model if some integrity constraints between elements of the frame are known to be true and must be considered in the fusion. DSmT allows to solve static or dynamic ${ }^{2}$ fusion problems in the same mathematical framework. For convenience, one denotes by $G^{\Theta}$ the generalized fusion space or generalized power set including integrity constraints (that is, exclusivity as well as possible nonexistence restrictions between some elements of $\Theta$ ), so that $G^{\Theta}=D^{\Theta}$ when no constraint enters in the model, or $G^{\Theta}=2^{\Theta}$ when one wants to work with Shafer's model (see [34] for details and examples), or $G^{\Theta}=\Theta$ when working with probability model. If one wants to work with the space closed under union $\cup$, intersection $\cap$, and complementarity C operators, then $G^{\Theta}=S^{\Theta}$, i.e. the superpower set (see next section). A more general introduction of DSmT can be found in Chapter 1 of [36].

In DSmT, the fusion of two sources of evidence characterized by $m_{1}(\cdot)$ and $m_{2}(\cdot)$ is defined by $m_{P C R 5}(\emptyset)=0$ and $\forall X \in G^{\Theta} \backslash\{\emptyset\}$

$$
m_{P C R 5}(X)=m_{12}(X)+\sum_{\substack{Y \in G^{\Theta} \\ X \cap Y=\emptyset}}\left[\frac{m_{1}(X)^{2} m_{2}(Y)}{m_{1}(X)+m_{2}(Y)}+\frac{m_{2}(X)^{2} m_{1}(Y)}{m_{2}(X)+m_{1}(Y)}\right]
$$

where all sets involved in the above formula are in the canonical form. $m_{12}(X) \equiv m_{\cap}(X)=\underset{\substack{X_{1}, X_{2} \in G^{\Theta} \\ X_{1} \cap X_{2}=X}}{m_{1}\left(X_{1}\right) m_{2}\left(X_{2}\right)} \underset{c o r r e-}{c}$ sponds to the conjunctive consensus on $X$ between $n=2$ sources and where all denominators are different from zero. If a denominator is zero, that fraction is discarded. A general formula of PCR5 for the fusion of $n>2$ sources has been proposed in [35].

\footnotetext{
2 i.e. when the frame or its model change with time.
} 


\subsection{Unification of fusion theory (UFT)}

Recently Smarandache has proposed in [33,35] an extension of DSmT by considering a super-power set $S^{\Theta}$ as the Boolean algebra on $\Theta$, that is, $S^{\Theta}=(\Theta, \cap, \cup, C(\cdot))$. In other words, $S^{\Theta}$ is assumed to be closed under union $\cup$, intersection $\cap$, and complement $C(\cdot)$ of sets respectively. With respect to the partial ordering relation, the inclusion $\subseteq$, the minimum element is the empty set $\emptyset$, and the maximal element is the total ignorance $I=\bigcup_{i=1}^{n} \theta_{i}$. Since it extends the power set space through the closed operation of $\cap, \cup$ and complement (.) operators, that is, UFT not only considers the nonexclusive situation among the elements, but also considers the exclusive, exhaustive, nonexhaustive situation, and even the open and closed world. Typically, when $\Theta=\{A, B\}$, in UFT one works with $m(\cdot)$ such that,

$$
m(A)+m(B)+m(A \cap B)+m(A \cup B)+m(C(A))+m(C(B))+m(C(A) \cup \complement(B))=1 .
$$

\section{Evidence Support Measure of Similarity (ESMS)}

Definition 3.1. Let's consider a discrete and finite frame $\Theta$ and the fusion space $G^{\Theta}$ including integrity constraints of the model associated with $\Theta$. The infinite set of basic belief assignments defined on $G^{\Theta}$ is denoted by $m_{G^{\Theta}}$. An Evidence Support Measure of Similarity (ESMS) of two (generalized) basic belief assignments $m_{1}(\cdot)$ and $m_{2}(\cdot)$ in $m_{G^{\Theta}}$ is the function $\operatorname{Sim}(\cdot, \cdot): m_{G^{\Theta}} \times m_{G^{\Theta}} \rightarrow[0,1]$ satisfying the following conditions:

(1) Symmetry: $\forall m_{1}(\cdot), m_{2}(\cdot) \in m_{G^{\Theta}}, \operatorname{Sim}\left(m_{1}, m_{2}\right)=\operatorname{Sim}\left(m_{2}, m_{1}\right)$;

(2) Consistency: $\forall m(\cdot) \in m_{G^{\Theta}}, \operatorname{Sim}(m, m)=1$;

(3) Nonnegativity: $\forall m_{1}(\cdot), m_{2}(\cdot) \in m_{G^{\theta}}, \operatorname{Sim}\left(m_{1}, m_{2}\right) \geqslant 0$.

This definition has been given in [16] and we focus on it from the point of view of belief function. We will say that $m_{2}(\cdot)$ is more similar to $m_{1}(\cdot)$ than $m_{3}(\cdot)$ if and only if $\operatorname{Sim}\left(m_{1}, m_{2}\right) \geqslant \operatorname{Sim}\left(m_{1}, m_{3}\right)$. The maximum degree of similarity is naturally obtained when both bba's $m_{1}(\cdot)$ and $m_{2}(\cdot)$ coincide, which is expressed by consistency condition (2). The equality $\operatorname{Sim}\left(m_{1}, m_{2}\right)=0$ must be obtained when bba's of two different sources are not assigned to the same focal elements, that is, whenever $m_{1}(\cdot)$ is focused on $X \in G^{\Theta}$, which is denoted $m_{1}^{X}(\cdot)$ and corresponds to $m_{1}(X)=1$, and $m_{2}(\cdot)$ is focused on $Y \in G^{\Theta}$, that is, $m_{2}(\cdot)=m_{2}^{Y}(\cdot)$ such that $m_{2}(Y)=1$, with $X \cap Y=\emptyset$.

Obviously, for any bba $m_{1}(\cdot) \in m_{G^{\Theta}}$ (which is a $\left|G^{\Theta}\right|$-dimensional vector) and any small positive real number $\epsilon$, there exists at least one bba $m_{2}(\cdot) \in m_{G^{\ominus}}$ for a given distance measure ${ }^{3} d(\cdot, \cdot)$ such that $d\left(m_{1}, m_{2}\right) \leqslant \epsilon$.

Definition 3.2. (Agreement of evidence): If there exist two basic belief assignments $m_{1}(\cdot)$ and $m_{2}(\cdot)$ in $m_{G^{\ominus}}$ such that for some distance measure $d(\cdot, \cdot)$, one has $d\left(m_{1}, m_{2}\right) \leqslant \epsilon$ with $\epsilon>0$, then $m_{1}(\cdot)$ and $m_{2}(\cdot)$ are said $\epsilon$-consistent with respect to the distance $d(\cdot, \cdot)$.

As we know, the smaller $\epsilon>0$ is, the closer the distance $d\left(m_{1}, m_{2}\right)$ between $m_{1}(\cdot)$ and $m_{2}(\cdot)$ is, that is, the more similar or consistent $m_{1}(\cdot)$ and $m_{2}(\cdot)$ are.

From the previous definitions, ESMS is regarded as an interesting measure for evaluating the degree of similarity between two sources. We propose to use ESMS as a preprocessing/thresholding technique to reduce the complexity of the combination of evidential sources by keeping in the fusion only the sources which are $\epsilon$-consistent. $\epsilon$ is actually a threshold parameter which has to be tuned by the system designer and which depends on the application and computational resources.

\section{Several possible ESMS}

In this section we propose several possibilities for choosing an ESMS function $\operatorname{Sim}(\cdot, \cdot)$ defined in the general fusion space $G^{\Theta}$, where all models, that is, probability, Dempster-Shafer (DS), Dezert-Smarandache (DSm) and Unification of Fusion may be chosen as the working model, however, the two evidential sources, when computing their similarity, must simultaneously work in the same model. The direct computation of their similarity between two sources respectively working in the different models is not significant.

\subsection{Euclidean ESMS function $\operatorname{Sim}_{E}\left(m_{1}, m_{2}\right)$}

Definition 4.1. Let $\Theta=\left\{\theta_{1}, \ldots, \theta_{n}\right\}(n>1), m_{1}(\cdot)$ and $m_{2}(\cdot)$ in $m_{G^{\Theta}}, X_{i}$ the ith (generic) element of $G^{\Theta}$ and $\left|G^{\Theta}\right|$ the cardinality of $G^{\Theta}$. The following Euclidean ESMS function can be defined ${ }^{4}$ :

\footnotetext{
${ }^{3}$ Here we donot specify the distance measure and keep it only as a generic distance. Actually $d(\cdot, \cdot)$ can be any distance measure. In practice, the Euclidean distance is often used.

${ }^{4}$ This is an extension of what is proposed in [13] for working in any fusion space, that is, in $G^{\Theta}=2^{\Theta}$, $G^{\Theta}=D^{\Theta}$, or $G^{\Theta}=S^{\Theta}$. See [36], Chapter 1 for details.
} 


$$
\operatorname{Sim}_{E}\left(m_{1}, m_{2}\right)=1-\frac{1}{\sqrt{2}} \sqrt{\sum_{i=1}^{\left|G^{\Theta}\right|}\left(m_{1}\left(X_{i}\right)-m_{2}\left(X_{i}\right)\right)^{2}}
$$

Theorem 4.1. $\operatorname{Sim}_{E}\left(m_{1}, m_{2}\right)$ defined in (8) is an ESMS function.

(See proof in Appendix)

\subsection{Jousselme ESMS function $\operatorname{Sim}_{J}\left(m_{1}, m_{2}\right)$}

Definition 4.2. Let $m_{1}(\cdot)$ and $m_{2}(\cdot)$ be two basic belief assignments in $m_{G^{\Theta}}$ provided by the sources of evidence $S_{1}$ and $S_{2}$. Assumed $^{5}$ a $\left|G^{\Theta}\right| \times\left|G^{\Theta}\right|$ positively definite matrix $D=\left[D_{i j}\right]$, where $D_{i j}=\left|X_{i} \cap X_{j}\right| /\left|X_{i} \cup X_{j}\right|$, with $X_{i}, X_{j} \in G^{\Theta}$. Then, Jousselme ESMS function can be redefined from the Jousselme et al. measure [13]:

$$
\operatorname{Sim}_{J}\left(m_{1}, m_{2}\right)=1-\frac{1}{\sqrt{2}} \sqrt{\left(m_{1}-m_{2}\right)^{T} D\left(m_{1}-m_{2}\right)}
$$

or equivalently

$$
\operatorname{Sim}_{J}\left(m_{1}, m_{2}\right)=1-\frac{1}{\sqrt{2}} \sqrt{m_{1}^{2}+m_{2}^{2}-2\left\langle m_{1}, m_{2}\right\rangle},
$$

where $\left\langle m_{1}, m_{2}\right\rangle$ is the scalar product defined as:

$$
\left\langle m_{1}, m_{2}\right\rangle=\sum_{i=1}^{\left|G^{\Theta}\right|} \sum_{j=1}^{\left|G^{\Theta}\right|} D_{i j} m_{1}\left(X_{i}\right) m_{2}\left(X_{j}\right),
$$

$X_{i}, X_{j} \in G^{\Theta}, i, j=1, \ldots, s,\left|G^{\Theta}\right|$.

Theorem 4.2. $\operatorname{Sim}_{J}\left(m_{1}, m_{2}\right)$ defined in formula (9) is an ESMS function.

(See proof in Appendix).

Actually $\operatorname{Sim}_{E}\left(m_{1}, m_{2}\right)$ is nothing but a special case of $\operatorname{Sim}_{J}\left(m_{1}, m_{2}\right)$ when taking $D$ as the $\left|G^{\Theta}\right| \times\left|G^{\Theta}\right|$ identity matrix.

\subsection{Ordered ESMS function $\operatorname{Sim}_{O}\left(m_{1}, m_{2}\right)$}

The definition of the (partial) ordered ESMS function $\operatorname{Sim}_{O}\left(m_{1}, m_{2}\right)$ is similar to $\operatorname{Sim}_{J}\left(m_{1}, m_{2}\right)$ but instead of using Jousselme's matrix $D=\left[D_{i j}\right]$, where $D_{i j}=\left|X_{i} \cap X_{j}\right| /\left|X_{i} \cup X_{j}\right|$, with $X_{i}, X_{j} \in G^{\Theta}$, we choose the DSm matrix $O=\left[O_{i j}\right]$, where $O_{i j}=s(-$ $\left.X_{i} \cap X_{j}\right) / s\left(X_{i} \cup X_{j}\right)$, and $s(X)$ corresponds to the intrinsic informational content of the proposition $X$ defined in details in [34] (Chapter 3). $s(X)$ is used for partially ordering the elements of $G^{\Theta}$. More precisely, $s(X)$ is the sum of the inverse of the length of the components of Smarandache's $\operatorname{code}^{6}$ of $X$.

As a simple example, let's take $\Theta=\left\{\theta_{1}, \theta_{2}\right\}$ with free DSm model (that is, when all elements are nonexclusive two-by-two), then the partially ${ }^{7}$ ordered hyper-power set $G^{\Theta}=D^{\Theta}$ is given by $D^{\Theta}=\left\{\emptyset, \theta_{1} \cap \theta_{2}, \theta_{1}, \theta_{2}, \theta_{1} \cup \theta_{2}\right\}$. As we know, $s(\emptyset)=0$, $s\left(\theta_{1} \cap \theta_{2}\right)=1 / 2, s\left(\theta_{1}\right)=1+1 / 2, s\left(\theta_{2}\right)=1+1 / 2$ and $s\left(\theta_{1} \cup \theta_{2}\right)=1+1+1 / 2$ since Smarandache's codes of $\emptyset, \theta_{1}, \theta_{2}, \theta_{1} \cap \theta_{2}$ and $\theta_{1} \cup \theta_{2}$ are respectively given by $\{\langle\cdot\rangle\}$ (empty code), $\{\langle 1\rangle,\langle 12\rangle\},\{\langle 2\rangle,\langle 12\rangle\},\{\langle 12\rangle\}$ and $\{\langle 1\rangle,\langle 12\rangle,\langle 2\rangle\}$. The matrix $O$ is defined by ${ }^{8}$

$$
O=\left[\begin{array}{llll}
\frac{s\left(\theta_{1} \cap \theta_{2}\right)}{s\left(\theta_{1} \cap \theta_{2}\right)} & \frac{s\left(\theta_{1} \cap \theta_{2}\right)}{s\left(\theta_{1}\right)} & \frac{s\left(\theta_{1} \cap \theta_{2}\right)}{s\left(\theta_{2}\right)} & \frac{s\left(\theta_{1} \cap \theta_{2}\right)}{s\left(\theta_{1} \cup \theta_{2}\right)} \\
\frac{s\left(\theta_{1} \cap \theta_{2}\right)}{s\left(\theta_{1}\right)} & \frac{s\left(\theta_{1}\right)}{s\left(\theta_{1}\right)} & \frac{s\left(\theta_{1} \cap \theta_{2}\right)}{s\left(\theta_{1} \cup \theta_{2}\right)} & \frac{s\left(\theta_{1}\right)}{s\left(\theta_{1} \cup \theta_{2}\right)} \\
\frac{s\left(\theta_{1} \cap \theta_{2}\right)}{s\left(\theta_{2}\right)} & \frac{s\left(\theta_{1} \cap \theta_{2}\right)}{s\left(\theta_{1} \cup \theta_{2}\right)} & \frac{s\left(\theta_{2}\right)}{s\left(\theta_{2}\right)} & \frac{s\left(\theta_{2}\right)}{s\left(\theta_{1} \cup \theta_{2}\right)} \\
\frac{s\left(\theta_{1} \cap \theta_{2}\right)}{s\left(\theta_{1} \cup \theta_{2}\right)} & \frac{s\left(\theta_{1}\right)}{s\left(\theta_{1} \cup \theta_{2}\right)} & \frac{s\left(\theta_{2}\right)}{s\left(\theta_{1} \cup \theta_{2}\right)} & \frac{s\left(\theta_{1} \cup X_{2}\right)}{s\left(\theta_{1} \cup \theta_{2}\right)}
\end{array}\right]=\left[\begin{array}{cccc}
1 & 1 / 3 & 1 / 3 & 1 / 5 \\
1 / 3 & 1 & 1 / 5 & 3 / 5 \\
1 / 3 & 1 / 5 & 1 & 3 / 5 \\
1 / 5 & 3 / 5 & 3 / 5 & 1
\end{array}\right] .
$$

It is easy to verify that $O$ is a positively definite matrix by checking the positivity of all its eigenvalues which are $\lambda_{1}=0.800>0, \lambda_{2} \approx 0.835>0, \lambda_{3} \approx 0.205>0$ and $\lambda_{4} \approx 2.160>0$ respectively. We have verified that $O$ is a positively definite matrix for $\operatorname{Card}(\Theta)=n \leqslant 5$. However, it is an open challenge to prove that $O$ is positively definite for any cardinality of $\Theta$

\footnotetext{
${ }^{5}$ Actually, Jousselme et al. in [13] did not prove that $D=\left[D_{i j}=\left|X_{i} \cap X_{j}\right| /\left|X_{i} \cup X_{j}\right|\right]$ is a positively definite matrix. $D$ is until now assumed to be positively definite. This is only a conjecture and proving it is an open challenge.

${ }^{6}$ Smarandache code is a representation of disjoint parts of the Venn diagram of the frame $\Theta$ under consideration. This code depends on the model for $\Theta$. For example, let's take $\Theta=\left\{\theta_{1}, \theta_{2}\right\}$. If $\theta_{1} \cap \theta_{2}=\emptyset$ (Shafer's model) is assumed, then the code of $\theta_{1}$ is $\langle 1\rangle$, whereas if $\theta_{1} \cap \theta_{2} \neq \emptyset$ (free DSm model) is assumed, then the code of $\theta_{1}$ will be $\{\langle 1\rangle,\langle 12\rangle$. The length of a component of a code is the number of characters between $\langle$ and $\rangle$ in Smarandache's notation. For example, the length of component $\langle 12\rangle$ is 2 . See [34], pp. 42-43 for details.

7 This is a partial order since $s\left(\theta_{1}\right)=s\left(\theta_{2}\right)$.

${ }^{8}$ Actually, one works with $G^{\Theta} \backslash\{\emptyset\}$, and thus the column and row corresponding to the empty set do not enter in the definition of 0 .
} 
greater than 5, and that's why we conjecture that $O$ is a positively definite matrix for $\operatorname{Card}(\Theta)>5$. Similarly to the formula (9), we define $\operatorname{Sim}_{O}\left(m_{1}, m_{2}\right)$ by:

$$
\operatorname{Sim}_{0}\left(m_{1}, m_{2}\right)=1-\frac{1}{\sqrt{2}} \sqrt{\left(m_{1}-m_{2}\right)^{T} O\left(m_{1}-m_{2}\right)} .
$$

It can be easily proved that $\operatorname{Sim}_{O}\left(m_{1}, m_{2}\right)$ is an ESMS function following a proof similar to the proof of Theorem 4.2 .

\subsection{ESMS function $\operatorname{Sim}_{B}\left(m_{1}, m_{2}\right)$}

Another ESMS function based on Bhattacharya's distance is defined as follows:

Definition 4.3. Let $m_{1}(\cdot), m_{2}(\cdot)$ be two basic belief assignments in $m_{G^{\Theta}}$, the ESMS function $\operatorname{Sim}_{B}\left(m_{1}, m_{2}\right)$ is defined by:

$$
\operatorname{Sim}_{B}\left(m_{1}, m_{2}\right)=1-\sqrt{1-\sum_{X_{i} \in \mathrm{F}} \sqrt{m_{1}\left(X_{i}\right) m_{2}\left(X_{i}\right)}},
$$

where $\mathrm{F}$ is the core of sources $S_{1}$ and $S_{2}$, i.e. the set of elements of $G^{\Theta}$ having a positive belief mass: $\mathrm{F}=\left\{X \in G^{\Theta} \mid m_{1}(X)>0\right.$ or $\left.m_{2}(X)>0\right\}$.

Theorem 4.3. $\operatorname{Sim}_{B}\left(m_{1}, m_{2}\right)$ defined in formula (11) is an ESMS function.

(See proof in Appendix).

\section{Comparison of ESMS functions}

In this section we analyze the performances of the four ESMS functions aforementioned through a simple example, where $\Theta=\left\{\theta_{1}, \theta_{2}, \theta_{3}\right\}$. We assume the free DSm model for $\Theta$. In such case, $G^{\Theta}=D^{\Theta}$ has 18 nonempty elements $a_{i}, i=1,2, \ldots, s, \ldots, 18$. $G^{\Theta}$ is closed under $\cap$ and $\cup$ operators according to Dedekind's lattice. Of course, we also may choose other lattice structures in a similar way according to the right model of $G^{\Theta}$.

Let's assume that $\theta_{2}$ is the true identity of the object under consideration. Its optimal belief assignment is denoted $m_{2}(\cdot) \triangleq\left\{m_{2}\left(\theta_{2}\right)=1, m_{2}(X)=0\right.$ for $\left.X \in G^{\Theta} \backslash\left\{\theta_{2}\right\}\right\}$. We perform a comparison of the four ESMS functions in order to show the evolution of the measure of similarity between $m_{1}(\cdot)$ and $m_{2}(\cdot)$ when $m_{1}(\cdot)$ varies from an equally distributed bba to $m_{2}(\cdot)$. More precisely, we start our simulation by choosing $m_{1}(\cdot)$ with all elements in $D^{\Theta}$ equally distributed, i.e. $m_{1}\left(a_{i}\right)=1 / 18$, for $i=1,2, \ldots, s, \ldots, 18$. Then, step-by-step we increase the mass of belief of $\theta_{2}$ by a constant increment $\Delta=0.01$ until reaching $m_{1}\left(\theta_{2}\right)=1$. Meanwhile, the masses $m_{1}(X)$ of belief of all elements $X \neq \theta_{2}$ of $G^{\Theta}$ take value [ $\left.1-m_{1}\left(\theta_{2}\right)\right] / 17$ to work with a normalized bba $m_{1}(\cdot)$. The basic belief mass assigned to empty set is always zero, that is, $m_{1}(\emptyset)=m_{2}(\emptyset)=0$.

The degree of similarity of the four ESMS functions are plotted in Fig. 1. The speed of convergence ${ }^{9}$ of a similarity measure is characterized by the slope angle $\alpha$ of the curve at origin, or by its tangent. Based on this speed of convergence criterion, the analysis of Fig. 1 yields the following remarks:

(1) According to Fig. $1, \tan \left(\alpha_{B}\right) \approx 0.86, \tan \left(\alpha_{E}\right) \approx 0.68, \tan \left(\alpha_{O}\right) \approx 0.6$ and $\tan \left(\alpha_{J}\right) \approx 0.57 . \operatorname{Sim}_{J}\left(m_{1}, m_{2}\right)$ has the slowest convergence speed, then $\operatorname{Sim}_{O}\left(m_{1}, m_{2}\right)$ takes second place.

(2) $\operatorname{Sim}_{E}\left(m_{1}, m_{2}\right)$ has a faster speed of convergence than $\operatorname{Sim}_{O}\left(m_{1}, m_{2}\right)$ and $\operatorname{Sim}_{J}\left(m_{1}, m_{2}\right)$ because it doesnot consider the intrinsic complexity of the elements in $G^{\Theta}$.

(3) The speed of convergence of $\operatorname{Sim}_{B}\left(m_{1}, m_{2}\right)$ is the fastest. When $m_{1}(\cdot)$ and $m_{2}(\cdot)$ become very $\operatorname{similar}, \operatorname{Sim}_{B}\left(m_{1}, m_{2}\right)$ becomes very quickly close to 1 . However, if a small dissimilarity between $m_{1}(\cdot)$ and $m_{2}(\cdot)$ occurs, then $\operatorname{Sim} m_{B}\left(m_{1}, m_{2}\right)$ becomes very small which actually makes it very sensitive to small dissimilarity perturbations.

(4) In summary, one sees that no definitive conclusion about the best choice among these four ESMS functions can be drawn in general. However if one considers the speed of convergence as the evaluation criterion for the choice of a dissimilarity measure, one sees that $\operatorname{Sim}_{B}\left(m_{1}, m_{2}\right)$ is the best choice, because it is very sensitive to small dissimilarity perturbations, whereas $\operatorname{Sim}_{J}\left(m_{1}, m_{2}\right)$ appears to be the worst choice with respect to such a criterion.

\section{Simulation results}

We present two simulation examples to show how ESMS filter performs within Generalized Fusion Machine (GFM), and to present its advantage.

\footnotetext{
${ }^{9}$ Here the convergence speed refers to how much the global agreement degree (similarity) is between $m_{1}(\cdot)$ and $m_{2}(\cdot)$ with the continuous decrease of $m_{1}\left(\theta_{2}\right)$.
} 


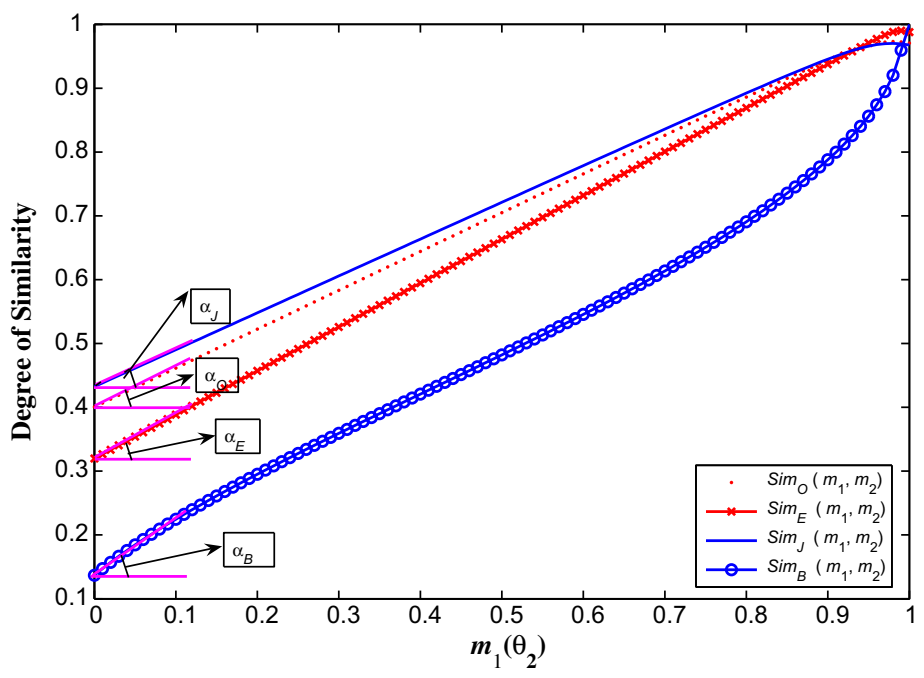

Fig. 1. Comparison of performance among ESMS functions.

Table 1

A list of given sources of evidence.

\begin{tabular}{|c|c|c|c|c|c|}
\hline$S$ & $m\left(\theta_{1}\right)$ & $m\left(\theta_{2}\right)$ & $m\left(\theta_{1} \cap \theta_{2}\right)$ & $m\left(\theta_{1} \cup \theta_{2}\right)$ & $\operatorname{Sim}_{E}$ \\
\hline$S_{1}$ & 0.3 & 0.4 & 0.2 & 0.1 & 0.8735 \\
\hline$S_{2}$ & 0.3 & 0.2 & 0.4 & 0.1 & 0.800 \\
\hline$S_{3}$ & 0.4 & 0.1 & 0.2 & 0.3 & 0.8268 \\
\hline$S_{4}$ & 0.7 & 0.1 & 0.1 & 0.1 & 0.7592 \\
\hline$S_{5}$ & 0.1 & 0.8 & 0.1 & 0.0 & 0.5550 \\
\hline$S_{6}$ & 0.5 & 0.2 & 0.2 & 0.1 & 0.9106 \\
\hline$S_{7}$ & 0.4 & 0.3 & 0.1 & 0.2 & 0.9368 \\
\hline$S_{8}$ & 0.3 & 0.1 & 0.2 & 0.4 & 0.7592 \\
\hline$S_{9}$ & 0.4 & 0.5 & 0.1 & 0.0 & 0.8103 \\
\hline$S_{10}$ & 0.8 & 0.1 & 0.0 & 0.1 & 0.6806 \\
\hline$S_{c 1}$ & 0.42 & 0.28 & 0.16 & 0.14 & 1.0000 \\
\hline$S_{11}$ & 0.5 & 0.0 & 0.2 & 0.3 & 0.7569 \\
\hline$S_{12}$ & 0.2 & 0.6 & 0.1 & 0.1 & 0.7205 \\
\hline$S_{13}$ & 0.4 & 0.3 & 0.2 & 0.1 & 0.9360 \\
\hline$S_{14}$ & 0.9 & 0.1 & 0.0 & 0.0 & 0.6230 \\
\hline$S_{15}$ & 0.5 & 0.2 & 0.1 & 0.2 & 0.9100 \\
\hline$S_{16}$ & 0.5 & 0.3 & 0.0 & 0.2 & 0.8900 \\
\hline$S_{17}$ & 0.5 & 0.0 & 0.1 & 0.4 & 0.7205 \\
\hline$S_{18}$ & 0.7 & 0.2 & 0.1 & 0.0 & 0.7807 \\
\hline$S_{19}$ & 0.1 & 0.7 & 0.1 & 0.1 & 0.6217 \\
\hline$S_{20}$ & 0.3 & 0.6 & 0.1 & 0.0 & 0.7390 \\
\hline$S_{c 2}$ & 0.44 & 0.29 & 0.13 & 0.14 & 1.0000 \\
\hline
\end{tabular}

Example 1. Let's take a 2D frame of discernment $\Theta=\left\{\theta_{1}, \theta_{2}\right\}$ and consider 20 equireliable sources of evidence according to Table 1 . We consider the free DSm model and the fusion space is the hyper-power set $D^{\Theta}=\left\{\theta_{1}, \theta_{2}, \theta_{1} \cap \theta_{2}, \theta_{1} \cup \theta_{2}\right\}$. $S_{c 1}$ denotes the barycentre of the front ten belief masses, while $S_{c 2}$ denotes the barycentre ${ }^{10}$ of all belief masses. The measure of similarity based on Euclidean ESMS function defined in Eq. (8) has been used here, but any other measures of similarity could be used instead. In this example, if we take 0.75 as the threshold value, we see from Table 1 and for the former 10 sources of evidence, that the measures of similarity of $S_{5}$ and $S_{10}$ with respect to $S_{c 1}$ are lower than 0.75 . Therefore, the sources $S_{5}$ and $S_{10}$ will be discarded/filtered in the fusion. If the threshold value is set to 0.8 , then the sources $S_{5}, S_{10}, S_{4}$ and $S_{8}$ will be discarded. That is, the higher the given threshold is, the fewer the number of information sources through the filter is.

The main steps of the algorithm for carrying out GFM are the following ones:

(1) Initialization of the parameters: the number of sources of evidence is set to zero, (that is, one has initially no source, $s=0$ ), so that the number of sources in the filter window is $n=0$.

\footnotetext{
${ }^{10}$ Let's denote $k=\left|G^{\Theta}\right|$ the cardinality of $G^{\Theta}$ and consider $S$ independent sources of evidence. If all sources are equireliable, the barycentre of belief masses of the $S$ sources is given by: $\forall j=1, \ldots, k, \bar{m}\left(X_{j}\right)=\frac{1}{S} \sum_{s=1}^{S} m_{s}\left(X_{j}\right)$, see [21] for details.
} 


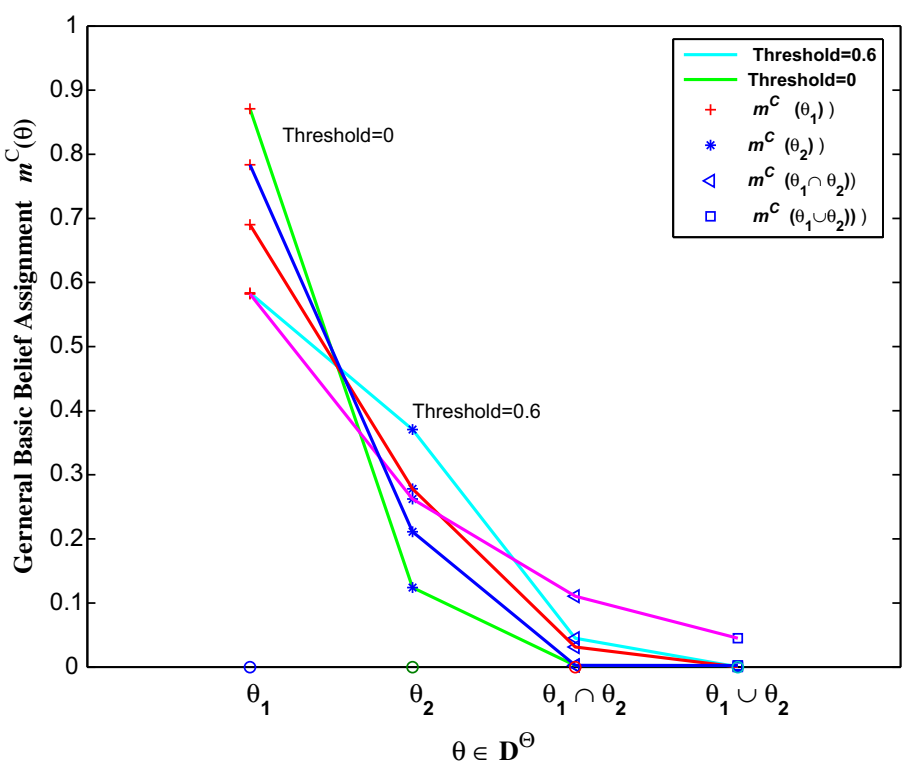

Fig. 2. Fusion result of the front 10 sources using different thresholds $(0 \sim 0.9)$.

(2) Include ${ }^{11}$ a source of evidence $\widehat{S}_{s}$ and then test if the number of sources $s$ is fewer than two. If $s \geqslant 2$, then go to the next step, otherwise include/take into account another source of evidence $\widehat{S}_{2}$.

(3) Based on the barycentre of gbba's of the front $n \leqslant 10$ evidence sources, the degrees of similarity are computed according to the formula (8), and compared with a prior tuned threshold. If it is larger than the threshold, then let $n=n+1$. Otherwise, introduce a new source of evidence $\widehat{S}_{s+1}$.

(4) If $n=1$, the current source, say $S$, is not involved in the fusion. If $n=2$, then the classical DSm rule [34] is applied in the fusion step between $S$ and $\widehat{S}_{2}$, that is, the conjunctive consensus. Then PCR5 rule [35] is applied to redistribute the remaining partial conflicts only to the sets involved in the corresponding partial conflicts. We get a new combined source affected with the same index $S$. If $2<n \leqslant 10$, after the current evidence source $\widehat{S}_{s}$ is combined with the final source of evidence produced last time, a new source of evidence is obtained and assigned to $S$ again. Whenever $n \leqslant 10$, go back to step (2), otherwise, the current source of evidence $\widehat{S}_{s}$ under test has been accepted by the ESMS filter, $\widehat{S}_{i}$ is assigned to $\widehat{S}_{i-1}, i \in[2, s, 10]$, and $\widehat{S}_{s}$ is assigned to $\widehat{S}_{10}$. Then, $\widehat{S}_{10}$ is combined with the last source $S$, the combined result is reassigned ${ }^{12}$ to $S$, and then, go back to step (2).

(5) Test whether to stop or not ${ }^{13}$ : if not, then introduce a new source of evidence $\widehat{S}_{s+1}$, otherwise stop and exit.

We show two simulation results in Figs. 2 and 3 following the working principle of GFM, when we use the sources of evidence listed in Table 1.

The comparison of Figs. 2 and 3 yields the following remarks:

(1) On Fig. 2, we donot see the real advantage of ESMS filter since the convergence to $\theta_{1}$ without ESMS filter (green curve) is better than with ESMS filter in terms of improving fusion precision. This is because some useful sources of information are filtered and thrown away with the increase of the threshold. Therefore the number of sources entering in the final fusion will decrease. This yields a slower speed of convergence to $\theta_{1}$. For example, let's consider the source $S_{3}$ in Table 1. If $S_{3}$ is combined with itself only once, according to formula (6), the fusion result is $S=\left[m_{N}\left(\theta_{1}\right)=0.5707\right.$, $\left.m_{N}\left(\theta_{2}\right)=0.0993, m_{N}\left(\theta_{1} \cap \theta_{2}\right)=0.1680, m_{N}\left(\theta_{1} \cup \theta_{2}\right)=0.1620\right]{ }^{14}$ If combined twice, the result is $S=[0.6765$, $0.0852,0.1429,0.0954]$. If combined thrice, then the result is $S=[0.7453,0.00693,0.1216,0.0638]$. The more the combinational times is, the nearer to $1 m_{N}\left(\theta_{1}\right)$ is, and the nearer to $0 m_{N}\left(\theta_{2}\right)$ is. Therefore, ESMS filter might also result in losing some useful information, while it filters some bad information.

(2) On Fig. 3, one sees the role played by ESMS filter. When there are highly conflicting sources, the result of the fusion will not converge if ESMS filter is not used. With the fine-tuning of ESMS threshold, the convergence becomes better and better because ESMS filter processes the fused information, and withdraws the sources which might cause the results to be incorrect or imprecise, so that it improves the fusion precision and correctness.

\footnotetext{
11 We assume the free DSm model and consider that the general basic belief assignments are given.

${ }^{12}$ In this work, we also use an ESMS filter window in a sliding mode.

${ }^{13}$ In our experiment, judge whether the mobile robot stops receiving sonar's data.

${ }^{14} m_{N}(\cdot)$ refers to the new generalized basic belief assignment.
} 


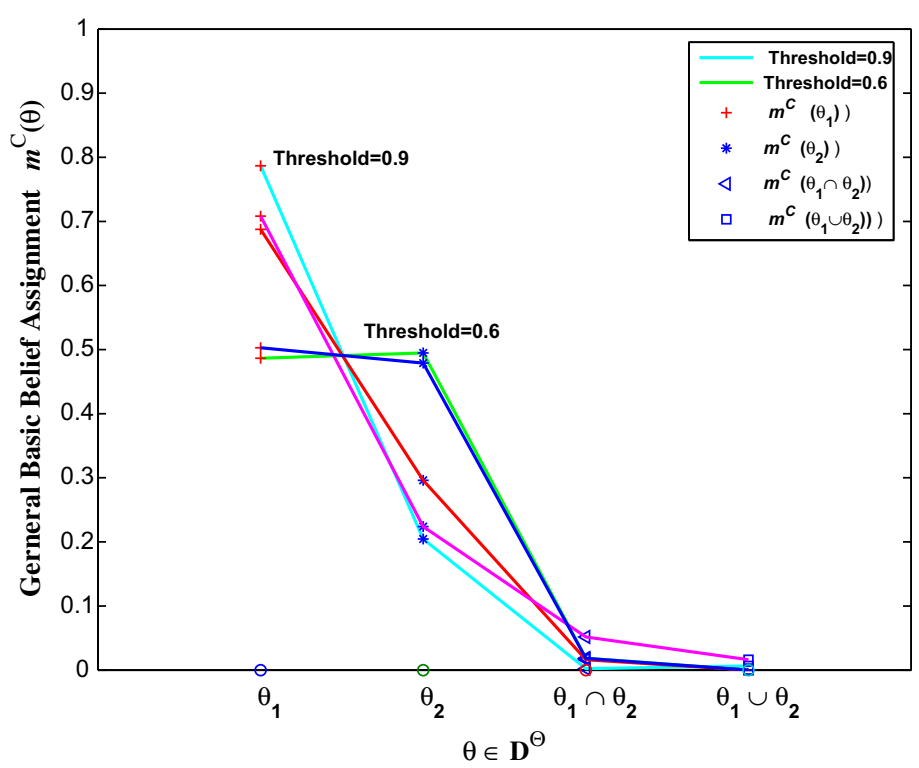

Fig. 3. Fusion result of the total 20 sources using different thresholds $(0 \sim 0.9)$.

(3) We increase the applicability of the classical rules of combination. Since we reduce the number of conflicting sources of evidence combined thanks to ESMS preprocessing, the degree of conflict between sources combined is kept low. Therefore, classical rules, like Dempster's rule, which do not perform well in high conflicting situations can also be applied in robot perception. Without ESMS preprocessing step, the classical fusion rules cannot work very well $[26,32]$. Therefore, we extend their domain of applicability when using ESMS filtering step.

Example 2. To show the advantage of the proposed method, we compare the performance of the method 1 (that is, using sequential fusion with PCR5 only) with the method 2 (using sequential fusion PCR5 with ESMS preprocessing) in terms of complexity cut. We consider the following two different cases:

- Case 1: (simple)

We consider the frame $\Theta=\left\{\theta_{1}, \theta_{2}\right\}$ with DSm model. There are four focal elements in $D^{\Theta} \backslash\{\emptyset\}$. We will consider 1001 sources of evidence having the following same belief assignments: $m_{s}\left(\theta_{1}\right)=0.4, m_{s}\left(\theta_{2}\right)=0.2, m_{s}\left(\theta_{1} \cap \theta_{2}\right)=0.3$, $m_{s}\left(\theta_{1} \cup \theta_{2}\right)=0.1,1 \leqslant s \leqslant 1001$.

- Case 2: (a bit more complex)

We consider a bit more complex case with $\Theta=\left\{\theta_{1}, \theta_{2}, \theta_{3}\right\}$ with DSm model. There are 18 elements in $D^{\Theta} \backslash\{\emptyset\}$. If all elements of $D^{\Theta} \backslash\{\emptyset\}$ are focal elements, then the computation of the combination becomes very costly and very time-consuming. We only consider 1001 sources of evidence having the following same belief assignments over partial elements in $D^{\Theta} \backslash\{\emptyset\}: m_{s}\left(\theta_{1}\right)=0.15, m_{s}\left(\theta_{2}\right)=0.25, m_{s}\left(\theta_{3}\right)=0.1, m_{s}\left(\theta_{1} \cap \theta_{2}\right)=0.05, m_{s}\left(\theta_{1} \cap \theta_{3}\right)=0.08, m_{s}\left(\theta_{2} \cap \theta_{3}\right)=0.18$, $m_{s}\left(\theta_{1} \cap \theta_{2} \cap \theta_{3}\right)=0.12, m_{s}\left(\theta_{1} \cup \theta_{2} \cup \theta_{3}\right)=0.07,1 \leqslant s \leqslant 1001$.

In either Case 1 or 2, all sources are the same. Of course, we had better produce a set of sources (1001) at random, and then partially choose sources by setting different ESMS thresholds. But a set of different sources (1001) produced at random are not easy to be listed here. Since all operations involved in each method do count in the computation time, that is, computation of the barycenter of belief masses, computation of ESMS, comparison and combination operations, obviously, there is nearly no difference between the 1001 same and different sources in term of computational burden. For convenience, here we replace the 1001 different sources with the 1001 same ones.

Table 2 shows, for Case 1, the comparison of the computing time ${ }^{15}$ for the sequential fusion based on PCR5 only ${ }^{16}$ (method 1) with the sequential fusion using PCR5 and ESMS preprocessor (method 2) when considering 21, 51 101, 201, 401, 501, 801 and 1001 sources of evidence selected. Similarly, Table 3 shows the comparison of computing time for Case 2.

The comparison of Tables 2 and 3 yields the following remarks:

\footnotetext{
15 using Processor: Intel (R) Core (TM)2 Duo CPU E7500 @ 2.93 GHz. EMS memory: 2.0 GB.

${ }^{16}$ That is without ESMS preprocessing.
} 
Table 2

Computing time with methods 1 and 2 for Case 1 .

\begin{tabular}{llllllllll}
\hline Number of sources & 1001 & 21 & 51 & 101 & 201 & 401 & 501 & 1001 & 233 \\
Time in $\mu$ s & 241 & 167 & 172 & 181 & 199 & 300 \\
Method used & 1 & 2 & 2 & 2 & 2 & 2 \\
\hline
\end{tabular}

Table 3

Computing time with methods 1 and 2 for Case 2 .

\begin{tabular}{|c|c|c|c|c|c|c|c|c|c|}
\hline Number of sources & 1001 & 21 & 51 & 101 & 201 & 401 & 501 & 801 & 1001 \\
\hline Time in $\mu$ s & 916 & 231 & 255 & 295 & 376 & 536 & 619 & 864 & 1025 \\
\hline Method used & 1 & 2 & 2 & 2 & 2 & 2 & 2 & 2 & 2 \\
\hline
\end{tabular}

(1) Even if we have introduced an ESMS preprocessing step (method 2), it turns out that finally a drastic cut of computing burden is obtained when these sources of evidence are highly conflicting and inconsistent. This is because we can significantly reduce the number of sources combined with ESMS criterion. Seen from Table 2 or Table 3, when 21 sources are chosen from 1001, that is, the remaining 800 sources assumed/regarded as highly conflicting and inconsistent sources (actually they are consistent absolutely, here it is only a hypothesis), the computing time is $167 \mu$ s in Table 2 and $231 \mu$ s in Table 3, whereas the computing time using method 1 is $241 \mu$ s in Table 2 and $916 \mu$ s in Table 3 . Therefore, we save $1-(167 / 241) \approx 30 \%$ of computing time in Table 2 when combining only 21 sources among 1001 , whereas we save $1-(231 / 916) \approx 75 \%$ of computing time in Table 3 .

(2) If the sources of evidence are less conflicting and more consistent, then ESMS preprocessing step seems useless. Indeed, one sees in Table 2 that the computing time using method 2 is $335 \mu$ s after combining 1001 sources, which is greater than the computing time of the method 1. The same remark holds for Case 2 as shown in Table 3 . In such a very particular case (when no source is filtered through ESMS), the computing time with method 2 becomes greater than that of method 1, because method 2 needs more computation to carry out ESMS preprocessing without gaining benefit (that is, saving computations) in PCR5 fusion step. Of course, with the increase of the number of focal elements, the computations for PCR5 fusion become more and more complex and the time taken by ESMS preprocessor becomes negligible with respect to PCR5 fusion step.

\section{An application in mobile robot perception}

The information acquired for building grid map using sonar sensors on a mobile robot is usually uncertain, imprecise and even highly conflicting. An application in autonomous robot perception and navigation provides a good platform to verify experimentally the advantage of ESMS filter in GFM. Although there exist many methods of building map based either on Probability Theory [39], FST (Fuzzy System Theory) [27], DST [37], GST (Grey System Theory) [42-44], or DSmT [23], we just compare the performances of the map building using a classical fusion machine without ESMS filter (i.e. CFMW) with the classical fusion machine with ESMS filter (called GFM) in DSmT framework only. A detailed comparison between our current ESMS-based approach with other methods is given in a companion paper in [24] where we show that ESMS-based approach outperforms other approaches using almost the same experimental conditions and inputs. In order to further lessen the measurement noises, we improve our past belief assignment model of sonar sensors in DSmT framework ${ }^{17}$ as follows:

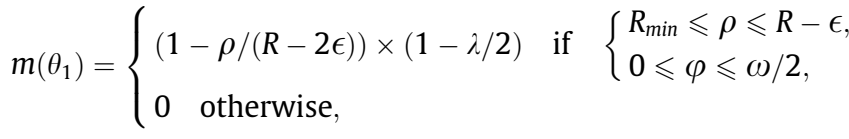

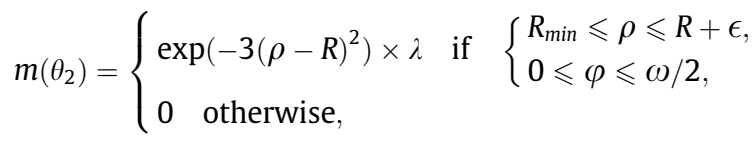

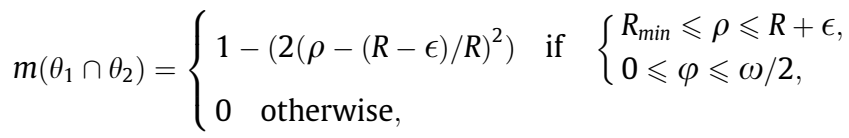

\footnotetext{
${ }^{17}$ We assume that there are only two focal elements $\theta_{1}$ and $\theta_{2}$ in the frame of discernment. Elements of hyper-power set are $\theta_{1}, \theta_{2}, \theta_{1} \cap \theta_{2}$ and $\theta_{1} \cup \theta_{2} . \theta_{1}$ represents the emptiness of a given grid cell, $\theta_{2}$ represents the occupancy for a given grid cell, $\theta_{1} \cap \theta_{2}$ means that there is some conflict between two sonar measurements for the same grid cell and $\theta_{1} \cup \theta_{2}$ represents the ignorance for a grid cell because of the possible lack of measurement.
} 


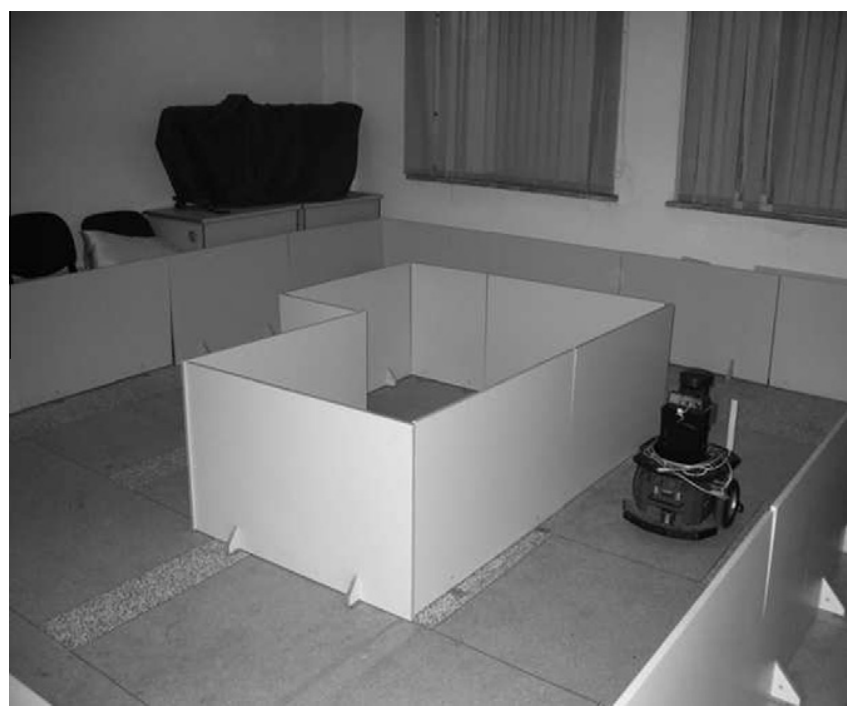

Fig. 4. The running robot in a real experimental environment.

$$
m\left(\theta_{1} \cup \theta_{2}\right)=\left\{\begin{array}{l}
\tan (2(\rho-R)) \times(1-\lambda) \quad \text { if } \quad R \leqslant \rho \leqslant R+\epsilon, \\
0 \quad \text { otherwise }
\end{array}\right.
$$

where, $\lambda$ is given by (see [8] for justification)

$$
\lambda=\left\{\begin{array}{l}
1-(2 \varphi / \omega)^{2} \\
0 \text { otherwise. }
\end{array}\right.
$$

The parameters $R, \rho, \epsilon, R_{\min }, \omega$, and $\varphi$ in formulas (12)-(16) were defined and used in [22-24]. $R$ is the sonar reading (measurement). $\rho$ is the distance between the grid cell and sonar's emitting point. $\epsilon$ is the measurement error. $R_{\min }$ is the minimal range of sonar sensors. $\omega$ is the scattering angle of sonar. $\varphi$ is the angle between the line (from the grid cell to sonar emitting point) and the sonar's emitting direction. The following functions $C_{1}$ and $C_{2}$ play an important role in lessening noises in the process of map building. $C_{1}$ function, proposed by Wang in [42-44], is a constriction function ${ }^{18}$ for sonar measurements defined by:

$$
C_{1}=\left\{\begin{array}{lll}
0 & \text { if } & \rho>\rho_{l_{2}}, \\
\frac{\rho_{l_{2}}-\rho}{\rho_{l_{2}}-\rho_{l_{1}}} & \text { if } & \rho_{l_{1}} \leqslant \rho \leqslant \rho_{l_{2}}, \\
1 & \text { if } & \rho<\rho_{l_{2}},
\end{array}\right.
$$

where, $\rho_{l_{1}}$ and $\rho_{l_{2}}$ represents the upper and lower limits of valid measurements, $C_{2}$ is the constraint function ${ }^{19}$ for sonar's uncertainty defined as follows:

$$
C_{2}=\left\{\begin{array}{lll}
\left(\frac{\rho-R+0.5 \epsilon}{0.5 \epsilon}\right)^{2} & \text { if } & \rho-R>-0.5 \epsilon \\
\left(\frac{\rho-R-0.5 \epsilon}{0.5 \epsilon}\right)^{2} & \text { if } & \rho-R<0.5 \epsilon, \\
0 & \text { if } & |\rho-R|>0.5 \epsilon
\end{array}\right.
$$

where, the product of $C_{1}$ and $C_{2}$ is multiplied by the belief assignment function $\left(m\left(\theta_{2}\right), m\left(\theta_{1} \cap \theta_{2}\right), m\left(\theta_{1} \cup \theta_{2}\right)\right)$ respectively.

The experiment is performed by running a Pioneer II mobile robot with 16 sonar detectors in the indoor laboratory environment as shown in Fig. 4. The environment's size is $4550 \mathrm{~mm} \times 3750 \mathrm{~mm}$. The environment is divided into $91 \times 75$ rectangular cells having the same size according to the grid map method. The robot starts to move from the location $(1 \mathrm{~m}, 0.6 \mathrm{~m})$, which faces towards 0 degrees. We take the left bottom corner as the global coordinate origin of the map. Objects/obstacles in the rectangular grid map are shown in Fig. 5. The processing steps of our intelligent perception and fusion system have been carried out with our software Toolbox developed under VC++ 6.0 and with OpenGL server as a client end. When the robot moves in the environment, the server end collects much information (such as the location of robot, sensors measurements, velocity) from the mobile robot and its sensors onboard. Through the protocol of TCP/IP, the client end can get any information from the server end and fuse them before displaying final result (the estimated grid map).

\footnotetext{
18 The main idea is that the sonar readings must be discounted according to sonar characteristics.

19 The main idea consists in assigning high belief assignments to sonar readings close to the sonar sensor.
} 


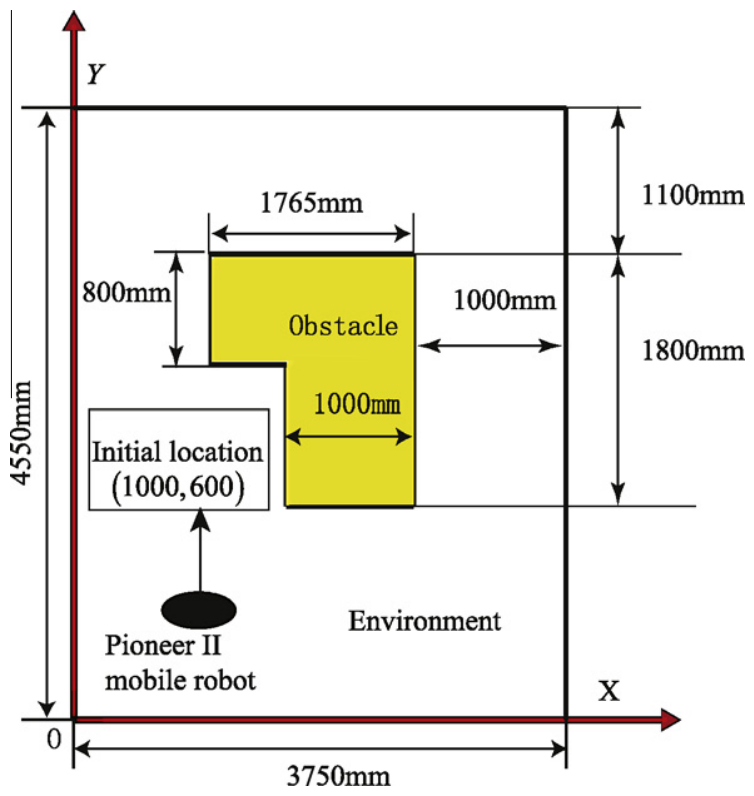

Fig. 5. Global coordinate system for the experiment.

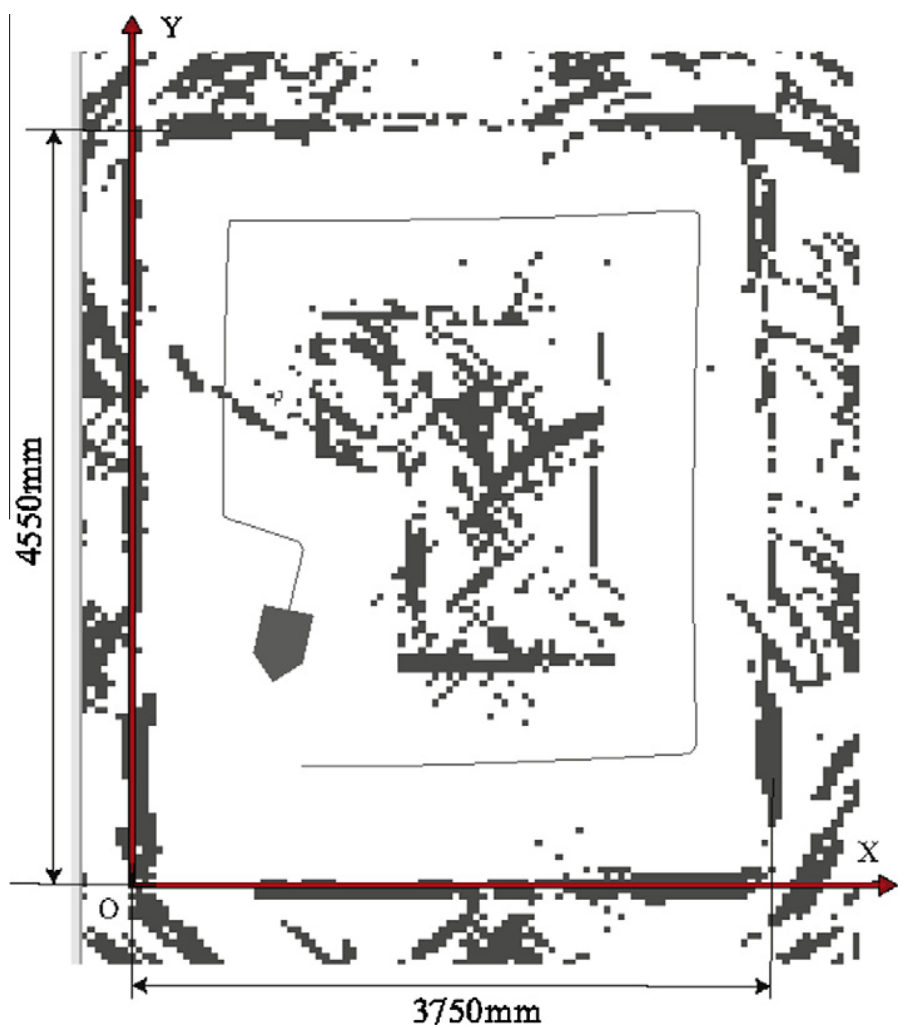

Fig. 6. Map building based on GFM before improving the sonar model.

Since our environment is small, the robot moves on a short distance during a relatively short period of time. Therefore, one only considers the self-localization method based on $\delta$-NFAM ${ }^{20}$ method $[20,22]$ with the search from $\theta-\delta_{\theta}$ to $\theta+\delta_{\theta}$. In

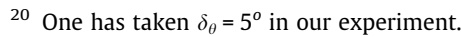




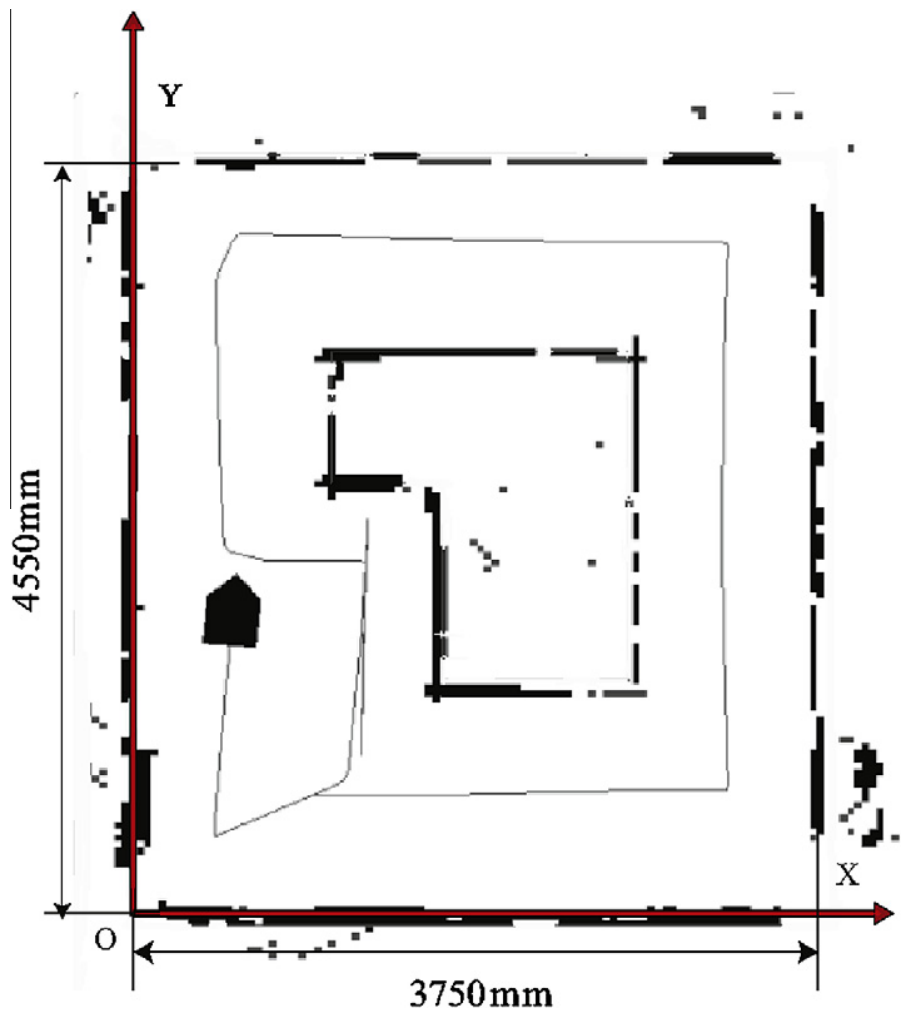

Fig. 7. Map building based on GFM after improving the sonar model.

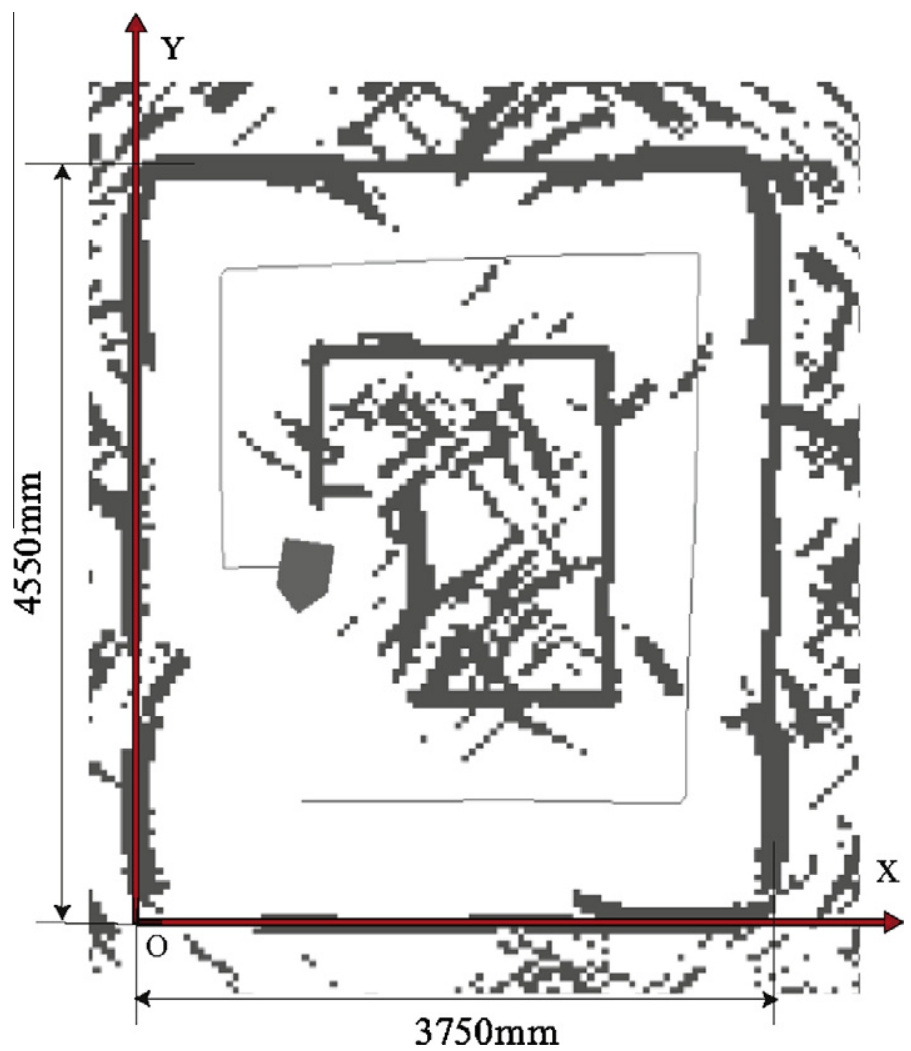

Fig. 8. Map building based on CFMW before improving the sonar model. 
order to reduce the computation burden, the restricted spreading arithmetic has been used. The main steps of map building with GFM are the following ones:

(1) Initialize the parameters of the robot (location, velocity, etc.).

(2) Acquire 16 sonar measurements, and robot's location from the odometer, when the robot is moving in the environment. The robot's pose is calibrated with our $\delta$-NFAM method [20,22] and the time clock with a 100 ms period is set up.

(3) Compute gbba of the fan-form area detected by each sonar sensor according to the formulas in [23].

(4) Apply DSmT-based GFM, that is, adopt Euclidean information filter to choose basic consistent sources of evidence according to the formula (8). Then combine the consistent sources with DSm conjunctive rule [3,4,34] and compute gbbas after combination. Then, redistribute partial conflicting masses to the gbba's of sets involved in the partial conflict only with PCR5 rule [35].

(5) Compute the belief of occupancy $\operatorname{Bel}\left(\theta_{2}\right)$ of some grid cells according to [34]. Save them into the map matrix and then go to step (6).

(6) Update the map of the environment (here we set the second timer, of whose interval is 100 ms). Generally speaking, the more the times of scanning map are, the more accurate the final map rebuilt is. At the same time, also test whether the robot stops receiving the sensed data: if yes, then stop fusion and exit, otherwise, go back to step (2).

In this experiment, we obtain the maps built by GFM before and after improving the sonar model as shown in Fig. 6 and 7 respectively. In order to show the advantage of ESMS filter in GFM, we also compare our approach with the classical fusion machine without ESMS filter (called CFMW). The maps built by CFMW before and after improving the sonar model are shown in Figs. 8 and 9 respectively. Whenever the map is built before or after improving the sonar model, one sees that GFM always outperforms CFMW because one obtains clearer boundary outlines and fewer noises in the map built. In addition, ESMS information filter coupled with PCR5 fusion rule, allows to reduce drastically the computational burden because ESMS filter can filter the outlier-sources. With GFM approach, only the most consistent sources of evidence are combined and this allows to reduce the uncertainty in the fusion result and to improve the robot perception of the surrounded environment.

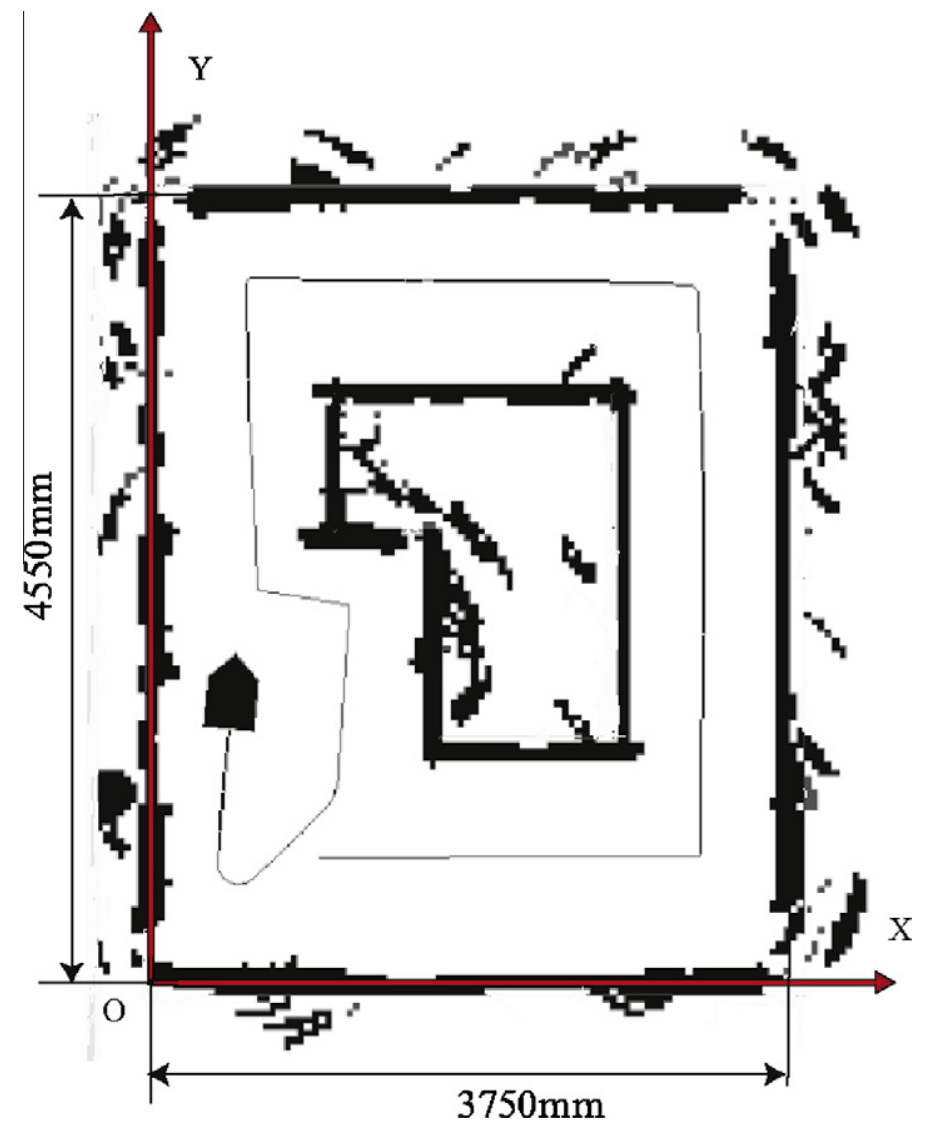

Fig. 9. Map building based on CFMW after improving the sonar model. 


\section{Conclusions}

In this paper, a general evidence supporting measure of similarity (ESMS) between two basic belief assignments has been proposed. ESMS can be used in different fusion spaces (lattice structures) and with different distance measures. This approach allows to select the most coherent subset of sources of evidence available and to reject outlier-sources which is seriously inconsistent with other sources. Therefore, a drastic cut of computational burden is possible with keeping good performances which is very attractive for real-time applications having limited computing resources. The hybrid of ESMS with the sophisticated and efficient PCR5 fusion rule of DSmT, called GFM (Generalized Fusion Machine), is specially useful and interesting in robotic applications involving real-time perception and navigation systems. The real application of GFM for mobile robot perception from sonar sensors presented in this work shows clearly a substantial improvement of the fusion result in map building/estimation of the surrounded environment. This work also shows an important role played by the most advanced fusion techniques for applications in robotics.

\section{Acknowledgments}

The authors are very grateful for Editor in Chief: Professor W. Pedrycz, Guest Editor: Professor Vassilis G. Kaburlasos and all anonymous reviewers who give us many valuable comments and suggestions and help us improve and present this paper. We are also very grateful for Charlyne Stein who helps us check and improve the linguistic quality of this paper.

\section{Appendix A}

Theorem 4.1. $\operatorname{Sim}_{E}\left(m_{1}, m_{2}\right)$ defined in (8) is an ESMS function.

\section{Proof}

(1) Let's prove that $\operatorname{Sim}_{E}\left(m_{1}, m_{2}\right) \in[0,1]$.If $\operatorname{Sim}_{E}\left(m_{1}, m_{2}\right)>1$, from (8) one would get $\frac{1}{\sqrt{2}} \sqrt{\sum_{i=1}^{\left|G^{\Theta}\right|}\left(m_{1}\left(X_{i}\right)-m_{2}\left(X_{i}\right)\right)^{2}}<0$ which is impossible, so that $\operatorname{Sim}_{E}\left(m_{1}, m_{2}\right) \leqslant 1$.Let's prove $\operatorname{Sim}_{E}\left(m_{1}, m_{2}\right) \geqslant 0$ or equivalently from $(8), \sum_{i=1}^{\left|G^{\Theta}\right|}\left(m_{1}\left(X_{i}\right)-m_{2}\left(X_{i}\right)\right)^{2} \leqslant$ 2.This inequality is equivalent to $\sum_{i=1}^{\left|G^{\Theta}\right|} m_{1}\left(X_{i}\right)^{2}+\sum_{i=1}^{\left|G^{\Theta}\right|} m_{2}\left(X_{i}\right)^{2} \leqslant 2+2 \sum_{i=1}^{\left|G^{\Theta}\right|} m_{1}\left(X_{i}\right) m_{2}\left(X_{i}\right)$. We denote it (i) for short.(i) always holds because one has $\left(\sum_{i=1}^{\left|G^{\Theta}\right|} m_{1}\left(X_{i}\right)^{2}+\sum_{i=1}^{\left|G^{\Theta}\right|} m_{2}\left(X_{i}\right)^{2}\right) \leqslant\left(\left[\sum_{i=1}^{\left|G^{\Theta}\right|} m_{1}\left(X_{i}\right)\right]^{2}+\left[\sum_{i=1}^{\left|G^{\Theta}\right|} m_{2}\left(X_{i}\right)\right]^{2}\right)$ and thus $\left(\sum_{i=1}^{\left|G^{\Theta}\right|} m_{1}\left(X_{i}\right)^{2}+\sum_{i=1}^{\left|G^{\Theta}\right|} m_{2}\left(X_{i}\right)^{2}\right) \leqslant 2$ because $\left[\sum_{i=1}^{\left|G^{\Theta}\right|} m_{s}\left(X_{i}\right)\right]^{2}=1$ for $s=1,2\left(m_{s}(\cdot)\right.$ being normalized bba).Therefore inequality (i) holds and thus $\operatorname{Sim}_{E}\left(m_{1}, m_{2}\right) \geqslant 0$.

(2) It is easy to check that $\operatorname{Sim}_{E}\left(m_{1}, m_{2}\right)$ satisfies the first condition of Definition 3.1 .

(3) If $m_{1}(\cdot)=m_{2}(\cdot)$, then $\operatorname{Sim}_{E}\left(m_{1}, m_{2}\right)=1$ because

$\sum_{i=1}^{\left|G^{\Theta}\right|}\left(m_{1}\left(X_{i}\right)-m_{2}\left(X_{i}\right)\right)^{2}=0$.

Thus the second condition of Definition 3.1 is also satisfied.

(4) Nonnegativity has been proven above in the first part. Herein we use a particular case to show that $\operatorname{Sim}(m 1, m 2)=0$, i.e. there exist $m_{1}^{X}$ and $m_{2}^{Y}$ for some $X, Y \in G^{\Theta} \backslash\{\emptyset\}$ such that $X \neq Y$, then according to (8), one gets $\sum_{i=1}^{\left|G^{\Theta}\right|}\left(m_{1}\left(X_{i}\right)-m_{2}\left(X_{i}\right)\right)^{2}=\left[m_{1}^{X}(X)\right]^{2}+\left[m_{2}^{Y}(Y)\right]^{2}=2$ and thus one has $\operatorname{Sim}_{E}\left(m_{1}^{X}, m_{2}^{Y}\right)=1-(\sqrt{2} / \sqrt{2})=0$, so that $\operatorname{Sim}_{E}(\cdot, \cdot)$ verifies the third condition of Definition 3.1.

Theorem 4.2. $\operatorname{Sim}_{J}\left(m_{1}, m_{2}\right)$ defined in formula (9) is an ESMS function.

\section{Proof}

(1) Since the matrix $D$ is conjectured to be a positively definite matrix, $\operatorname{Sim}_{J}\left(m_{1}, m_{2}\right)$ satisfies the condition of symmetry.

(2) If $m_{1}$ is equal to $m_{2}$, according to (9), one gets $\operatorname{Sim}_{J}\left(m_{1}, m_{2}\right)=1$. On the other hand, if $\operatorname{Sim}_{J}\left(m_{1}, m_{2}\right)=1$, then the condition $m_{1}=m_{2}$ holds. That is, the condition of consistency is satisfied.

(3) According to (9), it can be drawn that $\operatorname{Sim}_{J}\left(m_{1}, m_{2}\right) \leqslant \operatorname{Sim}_{E}\left(m_{1}, m_{2}\right)$, and since the minimum value of $\operatorname{Sim}_{J}\left(m_{1}, m_{2}\right)$ is zero, then $\operatorname{Sim}_{J}\left(m_{1}, m_{2}\right)$ is non-negative. 
(4) According to the definition of $\operatorname{Sim}_{J}\left(m_{1}, m_{2}\right)$, we can easily verify that $\operatorname{Sim}_{J}\left(m_{1}, m_{2}\right)$ is a true distance measure between $m_{1}$ and $m_{2}$.

Theorem 4.3. $\operatorname{Sim}_{B}\left(m_{1}, m_{2}\right)$ defined in formula (11) is an ESMS function.

\section{Proof}

(1) Since $\sum_{X_{i} \in \mathrm{F}} \sqrt{m_{1}\left(X_{i}\right) m_{2}\left(X_{i}\right)}=\sum_{X_{i} \in \mathrm{F}} \sqrt{m_{2}\left(X_{i}\right) m_{1}\left(X_{i}\right)}$, then $\operatorname{Sim}_{B}\left(m_{1}, m_{2}\right)$ satisfies the condition of symmetry.

(2) If $m_{1}(\cdot)=m_{2}(\cdot)$, according to (11),

$$
\sum_{X_{i} \in \mathrm{F}} \sqrt{m_{1}\left(X_{i}\right) m_{2}\left(X_{i}\right)}=\sum_{X_{i} \in \mathrm{F}} m_{1}\left(X_{i}\right)=1
$$

and therefore $\operatorname{Sim}_{B}\left(m_{1}, m_{1}\right)=1$. On the other hand, if $\operatorname{Sim}_{B}\left(m_{1}, m_{2}\right)=1$, then the condition $m_{1}(\cdot)=m_{2}(\cdot)$ holds. That is, the condition of consistency is satisfied.

(3) From the definition of bba, $\sum_{X_{i} \in \mathrm{F}} m_{1}\left(X_{i}\right)=1$. Therefore,

$$
\sum_{X_{i} \in \mathrm{F}} \sqrt{m_{1}\left(X_{i}\right) m_{2}\left(X_{i}\right)} \in[0,1] .
$$

According to (11), it can be drawn that $\operatorname{Sim}_{B}\left(m_{1}, m_{2}\right) \in[0,1]$; that is, the minimum value of $\operatorname{Sim}_{B}\left(m_{1}, m_{2}\right)$ is zero. Therefore, $\operatorname{Sim}_{B}\left(m_{1}, m_{2}\right)$ is a nonnegative measure.

(4) According to the definition of $\operatorname{Sim}_{B}\left(m_{1}, m_{2}\right)$, we can easily verify that $\operatorname{Sim}_{B}\left(m_{1}, m_{2}\right)$ is a true distance measure between $m_{1}$ and $m_{2}$.

\section{References}

[1] M. Bauer, Approximation algorithms and decision making in the Dempster-Shafer theory of evidence - an empirical study, Int. J. Approx. Reasoning 17 (2-3) (1997) 217-237.

[2] T.M. Cover, J.A. Thomas, Elements of Information Theory, Wiley, New York, 1991.

[3] J. Dezert, Foundations for a new theory of plausible and paradoxical reasoning, Int. J. Inform. Security 9 (2002) 3-57.

[4] J. Dezert, F. Smarandache, On the generation of hyper-power sets for the DSmT, in: Proceedings of 6th International Conference on Information Fusion, Cairns, Queensland, Australia, 2003, pp.1118-1125.

[5] J. Diaz, M. Rifqi, B. Bouchon-Meunier, A similarity measure between basic belief assignments, in: Proceedings of International Conference on Information fusion, Firenze, Italy, 2006, pp.1-8.

[6] R.P. Dilworth, Lattice theory, American Mathematical Society, Providence, Rhode Island, 1961.

[7] D. Dubois, H. Prade, Possibility, theory, probability theory and multiple-valued logics: a clarification, Ann. Math. Artif. Intell. 32 (2001) 35-66.

[8] A. Elfes, H. Moravec, High resolution maps from wide angle sonar, in: IEEE International Conference on Robotics and Automation, 1985, pp.116-121.

[9] G. Grätzer, General Lattice Theory, Academic Press, New York, 1978.

[10] ISIF (International Society of Information Fusion) website: <http://www.isif.org>.

[11] R. Jeffrey, The Logic of Decision, McGraw-Hill, New York, 1965.

[12] R. Jeffrey, Probability and the Art of Judgment, Cambridge University Press, Cambridge, 1992.

[13] A.-L. Jousselme, D. Grenier, E. Bossé, A new distance between two bodies of evidence, Inform. Fusion J. 2 (2001) 1-101.

[14] V.G. Kaburlasos, Towards a Unified Modeling and Knowledge-Representation Based on Lattice Theory - Computational Intelligence and Soft Computing Applications, series: Studies in Computational Intelligence, vol. 27, Springer, Heidelberg, Germany, 2006.

[15] V.G. Kaburlasos, I.N. Athanasiadis, P.A. Mitkas, Fuzzy lattice reasoning (FLR) classifier and its application for ambient ozone estimation, Int. J. Approx. Reason. 45 (1) (2007) 152-188.

[16] V.G. Kaburlasos, L. Moussiades, A. Vakali, Fuzzy lattice reasoning (FLR) type neural computation for weighted graph partitioning, Neurocomputing 72 (10-12) (2009) 2121-2133.

[17] W.B.S. Kandasamy, F. Smarandache, Basic Neutrosophic Algebraic Structures and Their Application to Fuzzy and Neutrosophic Models, Hexis, Church Rock, 2004.

[18] A.N. Kolmogorov, Foundations of the Theory of Probability, Chelsea Publishing Company, New York, 1960.

[19] S.E. Papadakis, V.G. Kaburlasos, Piecewise-linear approximation of nonlinear models based on probabilistically/possibilistically interpreted Intervals' Numbers (INs), Inform. Sci. 180 (24) (2010) 5060-5076.

[20] X. Li, Research on Fusion Method of Imperfect Information from Multi-source and Its Application, Ph. D. Thesis, Huazhong University of Science and Technology, China, 2007.

[21] X. Li, J. Dezert, X. Huang, Selection of sources as a prerequisite for information fusion with application to SLAM, in: Proceedings of the 9th International Conference on Information fusion, Florence, Italy, 2006, pp.10-13.

[22] X. Li,X. Huang, J. Dezert, Z. Wu, H.Zhang, Y. Xiong, DSmT-based generalized fusion machine for information fusion in robot map building, in: Proceedings of International Colloq. on Information Fusion 2007, Xi'an, China, 2007, pp. 63-70.

[23] X. Li, X. Huang, J. Dezert, L. Duan, M. Wang, A successful application of DSmT in sonar grid map building and comparison with DST-based approach, Int. J. Innovative Comput., Inf. Control 3 (3) (2007) 539-551.

[24] X. Li, B. Zhu, J. Dezert, X. Dai, An improved fusion machine for robot perception, Journal of intelligent and Robotic Systems, accepted for publication.

[25] R.H. Loschi, P.L. Iglesias, Jeffrey's rule: an alternative procedure to model uncertainty, Estadística $57(168,169)(2005) 11-26$.

[26] C.K. Murphy, Combining belief functions when evidence con flicts, Decision Support Syst. 29 (1) (2000) 1-9.

[27] C. Ofir, E. Yael, Adaptive fuzzy logic algorithm for grid-map based sensor fusion, in: IEEE Intell. Vehicles Symp., Parma, Italy, 2004, pp.625-630.

[28] A. Papoulis, Probability, Random Variables and Stochastic Processes, Revised 4th ed., McGraw Hill, 2002.

[29] S. Petit-Renaud, Application de la théorie des croyances et des systèmes flous à l'estimation fonctionnelle en présence d'informations incertaines ou imprécises, Ph.D. Thesis, Université de Technologie de Compiègne, France, December 1999.

[30] B. Ristic, Ph. Smets, Association of Uncertain Combat ID Declarations: The TBM Solution for Assignment Algorithms, in: Proceedings of Cogis '06 Conference, Paris, France, 2006, pp.1-8.

[31] B. Ristic, Ph. Smets, The TBM global distance measure for the association of uncertain combat ID declarations, Inform. Fusion 7 (3) (2006) $276-284$. 
[32] G. Shafer, A Mathematical Theory of Evidence, Princeton University Press, Princeton, NJ, 1976.

[33] F. Smarandache, Unification of fusion theories (UFT), Int. J. Appl. Math. Statist. 2 (2004) 1-14.

[34] F. Smarandache, J. Dezert (Editors), Advances and Applications of DSmT for Information Fusion (Collected works), American Research Press, Rehoboth, vol. 1, 2004. <http://www.gallup.unm.edu/ smarandache/DSmT-book1.pdf>.

[35] F. Smarandache, J. Dezert (Editors), Advances and Applications of DSmT for Information Fusion (Collected works), American Research Press, Rehoboth, vol. 2, 2006. <http://www.gallup.unm.edu/ smarandache/DSmT-book2.pdf>.

[36] F. Smarandache, J. Dezert (Editors), Advances and Applications of DSmT for Information Fusion (Collected works), American Research Press, Rehoboth, vol. 3, 2009. <http://www.gallup.unm.edu/ smarandache/DSmT-book3.pdf>.

[37] L. Su, Z. Cao, S. Wang, M. Tan, A real-time on-line method for exploring unknown environment with multiple robots, High Technol. Lett. 11 (2003) 5660.

[38] B. Tessem, Approximations for effecent computation in the theory of evidence, Artif. Intell. 61 (1993) 315-329.

[39] S. Thrun, D. Fox, W. Burgard, A probabilistic approach to concurrent mapping and localization for mobile robots, Mach. Learn. 31 (1-3) (1998) 9-53.

[40] A. Tversky, Features of similarity, Psychol. Rev. 84 (1977) 327-352.

[41] P. Walley, Statistical Reasoning with Imprecise Probabilities, Chapman and Hall, London, 1991.

[42] W. Wang, W. Chen, Y. Xi, Uncertainty sensor information processing in map building of mobile robot, Acta Automatica Sinica 29 (2) (2003) 267-274.

[43] W. Wang, Y. Xi, W. Chen, Map building for a mobile robot based on grey system theory, J. Syst. Eng. Elec. 14 (3) (2003) 67-72.

[44] W. Wang, Map building by mobile robots in unknown environment, Ph. D. Thesis, Shanghai Jiao Tong University, China, 2003.

[45] D. Wu, J. Mendel, A vector similarity measure for linguistic approximation: Interval type-2 and type-1 fuzzy sets, Inform. Sci. 178 (2) (2008) 381-402.

[46] D. Wu, J. Mendel, A comparative study of ranking methods, similarity measures and uncertainty measures for interval type-2 fuzzy sets, Inform. Sci. 179 (8) (2009) 1169-1192.

[47] X. Xin, T. Li, J. Lu, On derivations of lattices, Inform. Sci. 178 (2) (2008) 307-316.

[48] L. Zadeh, Fuzzy sets as the basis for a theory of possibility, Fuzzy Sets Syst. 1 (1978) 3-28.

[49] L.M. Zouhal, T. Denœux, An evidence-theoric $k$-NN rule with parameter optimization, IEEE trans. Syst. Man Cybern. C 28 (2) (1998) $263-271$. 